\title{
Representation Formulae of solutions of second order elliptic inequalities
}

\author{
Lorenzo D'Ambrosio $^{(1)}$, Enzo Mitidieri ${ }^{(2)}$
}

August 21, 2018

\begin{abstract}
We prove various representation formulas of solutions of second order differential inequalities on $\mathbb{R}^{N}$. As an outcome we obtain positivity results of the solutions of $-L u \geq 0$, where $L$ is a general second order elliptic operator in divergence form. Results of this type are very useful for proving related Liouville theorems of second order semilinear inequalities.
\end{abstract}

\section{Contents}

1 Introduction $\quad 1$

2 Main assumptions and examples 2

3 Representation Theorems $\quad 6$

3.1 Lemmata . . . . . . . . . . . . . . . . . . . . . 12

3.2 A priori estimates and the ring condition . . . . . . . . . . . . . . 19

3.3 Ring condition $(3.2)$ related to $\nabla u \ldots \ldots \ldots \ldots \ldots \ldots$

3.4 Proof of Theorem $6 \ldots \ldots \ldots \ldots \ldots \ldots \ldots$

3.5 Proof of Theorems 14 and $19 \ldots \ldots \ldots \ldots \ldots$

3.6 Additional remarks on the ring conditions . . . . . . . . . . . . 23

3.6.1 Ring condition and semilinear problems . . . . . . . . . . . 23

3.6.2 Other assumptions . . . . . . . . . . . . . . 25

4 Applications in the Carnot groups setting $\quad 26$

\section{Introduction}

The search of representation formulae of solutions of second order elliptic equations or inequalities has been an active research area over the past several years. See for instance the book [5] and the references therein for recent results and applications. Our goal in this paper is to find representation formulae of solution of problems of the type,

$$
-L u \geq 0 \quad \text { on } \quad \mathbb{R}^{N}
$$

Keywords. Representation formulae, Fundamental solution, sub-Laplacians in Carnot group. 2000 Mathematics Subject Classification. Primary 35H10, 35C15, 26D10. 
where $L$ is a general second order elliptic operator in divergence form (see section 2 below for the precise assumptions and fundamental properties) possessing a fundamental solution. In general if $u \in L_{l o c}^{1}\left(\mathbb{R}^{N}\right)$ is a distributional solution of $-L u \geq 0$, then $-L u:=\nu \geq 0$ is a nonnegative Radon measure, see [20]. One of the motivations for searching representation formulae, besides their intrinsic interest, relies on the fact that under suitable assumptions, nonnegative solutions of the problem,

$$
-L u=f(u) \quad \text { in } \quad \mathbb{R}^{N}
$$

where $f: \mathbb{R} \rightarrow \mathbb{R}_{+}$is a given continuous function, are indeed solutions of the integral equation

$$
u(x)=l+\int_{\mathbb{R}^{N}} \Gamma(x, y) f(u(y)) d y,
$$

where $l=\inf _{\mathbb{R}^{N}} u(x)$ and $\Gamma$ is a fundamental solution of $L$. See [8, 15] for some results in this direction.

When looking for Liouville theorems for (1.1), a typical simple statement can be formulated as follows. Let $L$ be a differential operator and let $f: \mathbb{R} \rightarrow \mathbb{R}$ be a given continuous function. Consider $u \in \Gamma\left(\mathbb{R}^{N}\right)$, where $\Gamma\left(\mathbb{R}^{N}\right)$ is a certain function class that depends on the differential operator $L$ and on the properties of $f$.

Liouville theorem. If $u$ is a nonnegative solution of (1.1) then $u \equiv$ constant in $\mathbb{R}^{N}$.

General Liouville theorems can be proved for several classes of semilinear/quasilinear equations and inequalities under some kind homogeneity assumptions on $L$. See [7, 10, 21] and the references therein for further information.

However, for operators $L$ with variable coefficients considered in this paper (see section 2 below), the approach based on the nonlinear capacity method developed in [21] needs to be modified. See 3.6.1. In the forthcoming paper [13] we shall prove that the representation formulae proved here are the suitable substitute of the nonlinear capacity method for proving Liouville theorems of (1.1).

Another motivation for searching representation formula of solution of (1.1) are the so called positivity results. These kind of results are related to the following fundamental question:

Positivity problem. When $f$ nonnegative implies that the possible solutions of (1.1) are positive?

Some contributions to this problem have been obtained in [15, 22] for some semilinear inequalities and in $[10,14]$ in the quasilinear context.

This paper is organised as follows. In Section 2 we state our main assumptions on the operator $L$ and we present some important examples. Section 3 is devoted to the formulation of the main results, while in subsection 3.1. we prove some necessary lemmata that we will use throughout the paper. In subsection 3.6.1 we comment and prove some results related to the ring condition, see (3.2) below. Finally section 4 is devoted to some applications in the Carnot group setting.

\section{Main assumptions and examples}

Let $A$ be an $N \times N$ matrix with entries $a_{i j} \in \mathscr{C}^{1}\left(\mathbb{R}^{N}\right)$. We shall deal with the operator $L$ of the form

$$
L(u):=\operatorname{div}(A \nabla u)=\sum_{i j=1}^{N} \frac{\partial}{\partial x_{i}}\left(a_{i j}(x) \frac{\partial u}{\partial x_{j}}\right) .
$$

Throughout this paper we shall suppose that the following conditions $H 1 \ldots H 7$. hold. 
H1. The matrix $A$ is semi-positive definite, that is:

$$
(A(x) \xi \cdot \xi) \geq 0 \text { for any } x, \xi \in \mathbb{R}^{N} .
$$

H2. For any $x \in \mathbb{R}^{N}$ there exists a fundamental solution $\Gamma_{x}=\Gamma(x, \cdot) \in L_{l o c}^{1}\left(\mathbb{R}^{N}\right)$ of $-L$. This means that $\Gamma: \mathbb{R}^{N} \times \mathbb{R}^{N} \backslash\left\{(x, y) \mid x=y \in \mathbb{R}^{N}\right\} \rightarrow \mathbb{R}$ is smooth and

$$
\int_{\mathbb{R}^{N}}-L(\phi)(y) \Gamma(x, y) d y=\phi(x)
$$

for any test function $\phi \in \mathscr{C}_{0}^{\infty}\left(\mathbb{R}^{N}\right)$. Denote with $A^{\prime}$ the transpose matrix of $A$ and with $L^{*}$ the adjoint operator of $L$. We have $L^{*}(u)=\operatorname{div}\left(A^{\prime} \nabla u\right)$ and $-L^{*}\left(\Gamma_{x}\right)=\delta_{x}$.

H3. $\lim _{y \rightarrow x} \Gamma(x, y)=+\infty$ for any $x \in \mathbb{R}^{N}$

H4. $\lim _{|y| \rightarrow \infty} \Gamma(x, y)=0$ for any $x \in \mathbb{R}^{N}$

H5. $\Gamma(x, y)>0$ for any $x \neq y$.

H6. For any $y \in \mathbb{R}^{N}, \Gamma(\cdot, y)$ is a fundamental solution of $-L^{*}$, that is $-L(\Gamma(\cdot, y))=\delta_{y}$.

H7. For any $x \in \mathbb{R}^{N}$ and $r>0$, we set

$$
\Omega_{r}(x):=\left\{y \in \mathbb{R}^{N} \mid \Gamma(x, y)>\frac{1}{r}\right\} \cup\{x\} .
$$

On the family $\Omega_{r}(x)$, we shall assume that

$$
\liminf _{\epsilon \rightarrow 0} \frac{\left|\Omega_{\epsilon}(x)\right|}{\epsilon}=0 \quad \text { for any } x \in \mathbb{R}^{N} .
$$

From the above assumptions on $\Gamma$, it is easy to see that

- For any $x \in \mathbb{R}$ and $r>0, \Omega_{r}(x)$ is a bounded, open set;

- For any $x \in \mathbb{R}$ and $r>0$, there exists $\epsilon>0$ such that $B(x, \epsilon) \subset \Omega_{r}(x)$;

- For any $x \in \mathbb{R}, \Omega_{r}(x)$ is a family of sets nondecreasing by inclusion such that $\Omega_{r}(x) \nearrow$ $\mathbb{R}^{N}($ as $r \rightarrow+\infty)$.

Notice that if $A$ is symmetric then assumption H6. implies $\Gamma(x, y)=\Gamma(y, x)$ and $L=L^{*}$. The above hypotheses are fulfilled in several concrete cases.

Example 1. Let $\mathcal{L}=\sum_{i=1}^{l} X_{i}^{2}$ where $X_{i}$ are smooth vector fields in $\mathbb{R}^{N}$ satisfying $H \ddot{o r}$ mander's condition of hypoellipticity:

$$
\operatorname{rank} \text { Lie }\left[X_{1}, \ldots, X_{l}\right]=N \text { at every } x \in \mathbb{R}^{N} .
$$

Assuming that: $X_{i}^{*}=-X_{i}$, where $X_{i}^{*}$ is the formal adjoint of $X_{i}$, there exists a symmetric semi-positive definite matrix $A$ such that $\mathcal{L}$ can be wtitten as $\mathcal{L} u=\operatorname{div}(A \nabla u)$.

In this setting hypotheses $[H 1.] \ldots[H 7$.$] and the properties on the family \Omega$ hold. See $[9,23]$ and the reference therein.

Further special cases is when it is possible to endow $\mathbb{R}^{N}$ with a Lie groups law and the vector fields $X_{i}$ are invariant by translation with respect to a such law. 
Example 2 (Carnot Groups). We begin quoting some preliminary results concerning Carnot groups (for more informations and proofs we refer the interested reader to [5, 16, 17]; see also the survey [19]). A Carnot group is a connected, simply connected, nilpotent Lie group $G$ of dimension $N \geq 2$ with graded Lie algebra $\mathcal{G}=V_{1} \oplus \cdots \oplus V_{r}$ such that $\left[V_{1}, V_{i}\right]=V_{i+1}$ for $i=1 \ldots r-1$ and $\left[V_{1}, V_{r}\right]=0$. A Carnot group $\mathbb{G}$ of dimension $N$ can be identified, up to an isomorphism, with the structure of homogeneous Carnot group $\left(\mathbb{R}^{N}, \circ, \delta_{\lambda}\right)$ defined as follows. We identify $\mathbb{G}$ with $\mathbb{R}^{N}$ endowed with a Lie group law $\circ$. We consider $\mathbb{R}^{N}$ splitted in $r$ subspaces $\mathbb{R}^{N}=\mathbb{R}^{n_{1}} \times \mathbb{R}^{n_{2}} \times \cdots \times \mathbb{R}^{n_{r}}$ with $n_{1}+n_{2}+\cdots+n_{r}=N$ and $\xi=\left(\xi^{(1)}, \ldots, \xi^{(r)}\right)$ with $\xi^{(i)} \in \mathbb{R}^{n_{i}}$. We shall assume that there exists a family of Lie group automorphisms, called dilation, $\delta_{\lambda}$ with $\lambda>0$ of the form $\delta_{\lambda}(\xi)=\left(\lambda \xi^{(1)}, \lambda^{2} \xi^{(2)}, \ldots, \lambda^{r} \xi^{(r)}\right)$. The Lie algebra of left-invariant vector fields on $\left(\mathbb{R}^{N}, \circ\right)$ is $\mathcal{G}$. For $i=1, \ldots, n_{1}=l$ let $X_{i}$ be the unique vector field in $\mathcal{G}$ that coincides with $\partial / \partial \xi_{i}^{(1)}$ at the origin. We require that the Lie algebra generated by $X_{1}, \ldots, X_{n_{1}}$ is the whole $\mathcal{G}$.

With the above hypotheses, we call $\mathbb{G}=\left(\mathbb{R}^{N}, \circ, \delta_{\lambda}\right)$ a homogeneous Carnot group. The canonical sub-Laplacian on $\mathbb{G}$ is the second order differential operator $\mathcal{L}=\sum_{i=1}^{l} X_{i}^{2}$. Let $Y_{1}, \ldots, Y_{l}$ be a basis of $\operatorname{span}\left\{X_{1}, \ldots, X_{l}\right\}$, the second order differential operator $\Delta_{G}=$ $\sum_{i=1}^{l} Y_{i}^{2}$ is called a sub-Laplacian on $\mathbb{G}$. We denote by $Q=\sum_{i=1}^{r} i n_{i}$ the homogeneous dimension of $\mathbb{G}$. In what follows we shall assume that $Q \geq 3$.

We shall list some properties and know results about Homogeneous Carnot groups.

The Lebesgue measure is the bi-invariant Haar measure. For any measurable set $E \subset \mathbb{R}^{N}$, we have $\left|\delta_{\lambda}(E)\right|=\lambda^{Q}|E|$. Since $Y_{1}, \ldots, Y_{l}$ generate the whole $\mathcal{G}$, any sub-Laplacian $\Delta_{G}$ satisfies the Hörmander's hypoellipticity condition. Moreover, the vector fields $Y_{1}, \ldots, Y_{l}$ are homogeneous of degree 1 with respect to $\delta_{\lambda}$.

In what follows we fix such vector fields $Y_{1}, \ldots, Y_{l}$ and when we shall refer to this setting we shall use the symbol symbol $\nabla_{L}$ to denote the vector field $\left(Y_{1}, \ldots, Y_{l}\right)$. As particular case of Example 1 there exists a symmetric semi-positive definite matrix $A$ such that $\Delta_{G}$ can be written as $\Delta_{G}=\operatorname{div}(A \nabla u)$.

A nonnegative continuous function $N^{*}: \mathbb{R}^{N} \rightarrow \mathbb{R}_{+}$is called a homogeneous norm on $\mathbb{G}$, if $N^{*}(\xi)=0$ if and only if $\xi=0$ and it is homogeneous of degree 1 with respect to $\delta_{\lambda}$ (i.e. $\left.N^{*}\left(\delta_{\lambda}(\xi)\right)=\lambda N^{*}(\xi)\right)$. We say that a homogeneous norm is symmetric if $N^{*}\left(\xi^{-1}\right)=N^{*}(\xi)$. A homogeneous norm $N^{*}$ defines on $\mathbb{G}$ a pseudo-distance defined as $d(\xi, \eta):=N^{*}\left(\xi^{-1} \circ \eta\right)$. For such function $d$, there holds only a pseudo-triangular inequality:

$$
d(\xi, \eta) \leq C d(\xi, \zeta)+C d(\zeta, \eta) \quad(\xi, \zeta, \eta \in \mathbb{G})
$$

with $C \geq 1$. Hence in general $d$, is not a distance. For a given homogeneous norm $N^{*}$, the symbol $B_{N^{*}}(\eta, R)$ denotes the ball $B_{N^{*}}(\eta, R):=\left\{\xi \in \mathbb{R}^{N} \mid N^{*}\left(\eta^{-1} \circ \xi\right)<R\right\}$. Then $\left|B_{N^{*}}(x, R)\right|=\left|B_{N^{*}}(0, R)\right|=c_{N^{*}} R^{Q}$.

If $N^{*}$ and $\tilde{N}$ are two homogeneous norms, then they are equivalent, that is, there exists a constant $C>0$ such that $C^{-1} N^{*}(\xi) \leq \tilde{N}(\xi) \leq C N^{*}(\xi)$.

Let $N^{*}$ be a homogeneous norm, then there exists a constant $C>0$ such that $C^{-1}|\xi| \leq$ $N^{*}(\xi) \leq C|\xi|^{1 / r}$, for $N^{*}(\xi) \leq 1$ and $|\cdot|$ stands for the Euclidean norm. An example of symmetric homogeneous norm is the following

$$
N_{S}(\xi):=\left(\sum_{i=1}^{r}\left|\xi_{i}\right|^{2 r ! / i}\right)^{1 / 2 r !} .
$$

Notice that if $N^{*}$ is a homogeneous norm differentiable a.e., then $\left|\nabla_{L} N^{*}\right|$ is homogeneous of degree 0 with respect to $\delta_{\lambda}$, hence $\left|\nabla_{L} N^{*}\right|$ is bounded. 
In [16] it is proved that for any sub-Laplacian $\Delta_{G}$ there exists a homogeneous symmetric norm $N_{2}$ on $\mathbb{G}$, often called gauge, such that $\Gamma_{\eta}(\xi):=\left(N_{2}\left(\eta^{-1} \circ \xi\right)\right)^{2-Q}$ is a fundamental solution of $-\Delta_{G}$ at $\eta$ (see also [5]). A such homogeneous norm (by hypoellipticity of $-\Delta_{G}$ ) is smooth off of the origin. We shall denote by $\psi_{\eta}$ the quantity $\psi_{\eta}:=\left|\nabla_{L} N_{2}\left(\eta^{-1} \circ \cdot\right)\right|$. Since $Y_{i} N_{2}$ is homogeneous of degree 0 with respect to $\delta_{\lambda}$, we have $\left\|\psi_{\eta}\right\|_{\infty}=\left\|\psi_{0}\right\|_{\infty}$ by left invariance of $Y_{i}$.

With the symbols used at point H7. above we have that $\Omega_{r}(x)=\left\{y \in \mathbb{R}^{N} \mid N_{2}\left(x^{-1} \circ y\right)<\right.$ $\left.r^{\frac{1}{Q-2}}\right\}$ and hence $\left|\Omega_{r}(x)\right|=\left|B_{N_{2}}\left(r^{\frac{1}{Q-2}}\right)\right|=C r^{\frac{Q}{Q-2}}$.

In what follows a relevant role will be played by the quantity $\frac{\left(\nabla \Gamma_{\eta} \cdot A \nabla \Gamma_{\eta}\right)}{\Gamma_{\eta}}$, which in the Carnot group setting can be rewritten as $(Q-2)^{2} \frac{\psi_{\eta}^{2}(\xi)}{N_{2}^{Q}\left(\eta^{-1} \circ \xi\right)}=(Q-2)^{2} \frac{\psi_{0}^{2}}{N_{2}^{Q}}\left(\eta^{-1} \circ \xi\right)$.

Finally, we remind that in the Carnot group setting a Liouville theorem holds. Namely if $\mathcal{L}$ is a sublaplacian then any $\mathcal{L}$-harmonic nonnegative function is constant. See $[4,5]$.

Example 3. Simple examples of Carnot groups are the usual Euclidean spaces $\mathbb{R}^{Q}$. Moreover, if $Q \leq 3$ then $\mathbb{G}$ is the ordinary Euclidean space $\mathbb{R}^{Q}$.

The simplest nontrivial example of a Carnot group is the Heisenberg group $\mathbb{H}^{1}=\mathbb{R}^{3}$. For an integer $n \geq 1$, the Heisenberg group $\mathbb{H}^{n}$ is defined as follows. Let $\xi=\left(\xi^{(1)}, \xi^{(2)}\right)=$ $\left(x_{1}, \ldots, x_{n}, y_{1}, \ldots, y_{n}, t\right)=(x, y, t) \in \mathbb{R}^{2 n} \times \mathbb{R}$. The Heisenberg group $\mathbb{H}^{n}$ is the set $\mathbb{R}^{2 n+1}$ endowed with the group law

$$
\hat{\xi} \circ \tilde{\xi}:=\left(\hat{x}+\tilde{x}, \hat{y}+\tilde{y}, \hat{t}+\tilde{t}+2 \sum_{i=1}^{n}\left(\tilde{x}_{i} \hat{y}_{i}-\hat{x}_{i} \tilde{y}_{i}\right)\right) .
$$

For $i=1, \ldots, n$, consider the vector fields

$$
X_{i}:=\frac{\partial}{\partial x_{i}}+2 y_{i} \frac{\partial}{\partial t}, Y_{i}:=\frac{\partial}{\partial y_{i}}-2 x_{i} \frac{\partial}{\partial t},
$$

and the associated Heisenberg gradient as follows $\nabla_{H}:=\left(X_{1}, \ldots, X_{n}, Y_{1}, \ldots, Y_{n}\right)$. The Kohn-Laplacian $\Delta_{H}$ is then the operator defined by $\Delta_{H}:=\sum_{i=1}^{n} X_{i}^{2}+Y_{i}^{2}$. The family of dilation is given by $\delta_{\lambda}(\xi):=\left(\lambda x, \lambda y, \lambda^{2} t\right)$. In $\mathbb{H}^{n}$ is defined the homogeneous norm

$$
|\xi|_{H}:=\left(\left(\sum_{i=1}^{n} x_{i}^{2}+y_{i}^{2}\right)^{2}+t^{2}\right)^{1 / 4} .
$$

The homogeneous dimension is $Q=2 n+2$ and the fundamental solution of the sub-Laplacian $-\Delta_{H}$ at point $\eta$ is given by $\Gamma_{\eta}(\xi)=N_{2}^{2-Q}\left(\eta^{-1} \circ \xi\right)=c\left|\eta^{-1} \circ \xi\right|_{H}^{-2 n}$.

Example 4. As particular case of the Example 1 is when the vector fields satisfying some further conditions. Namely, let $\left\{X_{1}, \ldots, X_{l}\right\}$ be a fixed set of linearly independent smooth vector fields on Euclidean space $\mathbb{R}^{N}$, satisfying the following properties:

- There exits a family of non-isotropic diagonal maps $\delta_{\lambda}$ of the form

$$
\delta_{\lambda}(x)=\left(\lambda^{\sigma_{1}} x_{1}, \ldots, \lambda^{\sigma_{N}} x_{N}\right) \quad \text { with } \quad 1=\sigma_{1} \leq \cdots \leq \sigma_{N} .
$$

We assume that such that $X_{1}, \ldots, X_{l}$ are $\delta_{\lambda}$-homogeneous of degree 1 with respect to the family of non-isotropic dilations $\left\{\delta_{\lambda}\right\}_{\lambda \geq 0}$.

- $X_{1}, \ldots, X_{l}$ satisfy Hörmander's rank condition at 0, i.e.,

$$
\operatorname{dim}\left\{X(0): X \in \operatorname{Lie}\left\{X_{1}, \ldots, X_{l}\right\}\right\}=N .
$$


$\bullet \sum_{j} \sigma_{j}>2$.

In this case by a lifting technique in [3] the authors proved that for the operator $\mathcal{L}=\sum_{i=1}^{l} X_{i}^{2}$ all the hypotheses $[H 1.] \ldots[H 7$.$] are fulfilled. We emphasise that even the$ hypothesis that $\Gamma$ vanishes at infinity is satisfied.

More precisely they show the existence of an homogeneous Carnot group $\left(\mathbb{R}^{N+p}, \circ, \tilde{\delta}\right)$ and vector fields $Z_{1}, \ldots, Z_{l}$, such that $\sum_{i=1}^{l} Z_{i}^{2}$ is a sub-Laplacian on $\mathbb{R}^{N+p}$ and the projection of $Z_{i}$ on $\mathbb{R}^{N}$ is $X_{i}(i=1, \ldots, l)$.

This last remark implies that for the operator $\mathcal{L}=\sum_{i=1}^{l} X_{i}^{2}$ Liouville theorem holds, that is any $\mathcal{L}$-harmonic nonnegative function is constant $^{1}$.

Example 5. The Grushin operator

$$
\partial_{x}^{2}+x^{2} \partial_{y}^{2}
$$

acting on $\mathbb{R}^{2}$, satisfies our assumptions $[H 1.] \ldots[H 7$.$] . Indeed this operator is of the type$ described in the Example 4. See also the seminal paper [18] and the references in [3].

The Grushin type operator

$$
\partial_{x}^{2}+x^{4} \partial_{y}^{2}
$$

is of the same type, see [3]. Our results apply to this operator too.

For further generalisations of these operators (dealing with several variables, as well as several weights), the explicit fundamental solutions have been computed in [2].

As a final example taken from [3], we remark that our results apply to the following Engel-type operator where the vector fields acting on $\mathbb{R}^{3}$ are given by

$$
X_{1}=\partial_{x_{1}} ; \quad X_{2}=x_{1} \partial_{x_{2}}+x_{1}^{2} \partial_{x_{3}} .
$$

\section{Representation Theorems}

Let $L$ be an operator of type (2.1) satisfying the assumptions $H 1 \ldots H 7$. of Section 2 . In this section we study the solutions of the inequality

$$
-L(u) \geq 0 \quad \text { on } \quad \mathbb{R}^{N} .
$$

If $u \in L_{l o c}^{1}\left(\mathbb{R}^{N}\right)$ is a distributional solution of (3.1) it follows that $-L(u)$ is in general a distribution. In our case it is a nonnegative distribution, therefore it is, actually, a nonnegative Radon measure. See [20]. Consequently, in general we may assume that $-L(u)=: \nu$ is a nonnegative Radon measure.

The following representation theorem of the solutions of (3.1) has its roots in [15].

Theorem 6 (Riesz representation). Let $u \in \mathscr{C}^{2}\left(\mathbb{R}^{N}\right)$ be such that $-L(u)=\nu \geq 0$.

A. Let $x \in \mathbb{R}^{N}$ and $l_{x} \in \mathbb{R}$ be such that

$$
\liminf _{R \rightarrow+\infty} \int_{\Omega_{2 R}(x) \backslash \Omega_{R}(x)} \frac{\left(\nabla \Gamma_{x} \cdot A \nabla \Gamma_{x}\right)}{\Gamma_{x}}\left(u-l_{x}\right) d y=0 .
$$

Then

$$
u(x)=l_{x}+\int_{\mathbb{R}^{N}} \Gamma(x, y) \nu(y) d y .
$$

Assume that for any $x \in \mathbb{R}^{N}$ (3.2) holds and set $l(x):=l_{x}$. Then

\footnotetext{
${ }^{1}$ Indeed if $u$ us a nonnegative function such that $\mathcal{L} u=0$, then by lifting $\tilde{\mathcal{L}} u=\sum_{i=1}^{l} Z_{i}^{2} u=0$, and since $\tilde{\mathcal{L}}$ is a sub-Laplacian, it follows that $u$ is constant.
} 
(a) $\inf u=\inf l$ (finite or not), $\sup u \geq \sup l$ (finite or not), and the following alternative holds,

$$
\text { either } u(x)>l(x), \forall x \in \mathbb{R}^{N}, \quad \text { or } \quad u \equiv l \text { and } \nu \equiv 0 \text {. }
$$

(b) If $w(\cdot):=\int_{\mathbb{R}^{N}} \Gamma(\cdot, y) \nu(y) d y \in L_{l o c}^{1}\left(\mathbb{R}^{N}\right)$ or equivalently $l \in L_{l o c}^{1}\left(\mathbb{R}^{N}\right)$, then the function $l$ is a distributional solution of

$$
-L(h)=0, \quad \text { on } \quad \mathbb{R}^{N} .
$$

Moreover if $\nu \not \equiv 0$, then there exists a constant $C>0$ such that

$$
\liminf _{R \rightarrow+\infty} R \int_{\Omega_{2 R}(x) \backslash \Omega_{R}(x)} \frac{\left(\nabla \Gamma_{x} \cdot A \nabla \Gamma_{x}\right)}{\Gamma_{x}}\left(u-l_{x}\right) d y \geq C>0,
$$

where $C$ depends on $\nu$ and it is independent on $u$ nor to the particular structure of the operator $L$, namely if $\nu \not \equiv 0$ on the set $\Omega_{s}(o)$ for some $s>0$ and $o \in \mathbb{R}^{N}$ then

$$
C=M \int_{\Omega_{s}(o)} \nu(y) d y
$$

where $M$ is a universal constant.

(c) if $u \geq 0$, then $w \in L_{l o c}^{1}\left(\mathbb{R}^{N}\right)$.

(d) Finally, if $l_{x}$ does not depends on $x, l_{x}=l \in \mathbb{R}$, then for any $x \in \mathbb{R}^{N}$

$$
u(x)=l+\int_{\mathbb{R}^{N}} \Gamma(x, y) \nu(y) d y .
$$

B. If $u$ is bounded from below then (3.2) is fulfilled for any $x \in \mathbb{R}^{N}$. Hence the claims in A hold true.

C. If there exists a function $h \in \mathscr{C}^{2}\left(\mathbb{R}^{N}\right)$ such that $L(h)=0$ and

$$
u(x)=h(x)+\int_{\mathbb{R}^{N}} \Gamma(x, y) \nu(y) d y,
$$

then for any $x \in \mathbb{R}^{N}$,

$$
h(x)=\frac{1}{\ln 2} \liminf _{R \rightarrow+\infty} \int_{\Omega_{2 R}(x) \backslash \Omega_{R}(x)} \frac{\left(\nabla \Gamma_{x} \cdot A \nabla \Gamma_{x}\right)}{\Gamma_{x}} u .
$$

Remark 7. We notice, see (3.36) below, that condition (3.2) is equivalent to require that

$$
l_{x}=\frac{1}{\ln 2} \liminf _{R \rightarrow+\infty} \int_{\Omega_{2 R}(x) \backslash \Omega_{R}(x)} \frac{\left(\nabla \Gamma_{x} \cdot A \nabla \Gamma_{x}\right)}{\Gamma_{x}} u
$$

exists and it is finite. See also Remark 10 below.

The next result deals with an assumption on the gradient of $\nabla u$ assuring that (3.2) is satisfied. For additional results of this type see Lemma 29 below. 
Theorem 8. Let $x \in \mathbb{R}^{N}$ and $u \in \mathscr{C}^{2}\left(\mathbb{R}^{N}\right)$ be such that $-L(u)=\nu \geq 0$. Assume that $\left|A^{\prime} \nabla \Gamma\right| \in L_{l o c}^{1}\left(\mathbb{R}^{N}\right)$.

If

$$
\liminf _{r \rightarrow+\infty} \int_{\Omega_{r}(x)}\left(\nabla \Gamma_{x} \cdot A \nabla u\right) d y<+\infty,
$$

then there exists $l_{x} \in \mathbb{R}$ such that (3.2) is satisfied and (3.3) holds. Moreover, we have

$$
\lim _{r \rightarrow+\infty} \int_{\Omega_{r}(x)}\left(\nabla \Gamma_{x} \cdot A \nabla u\right) d y=\int_{\mathbb{R}^{N}} \Gamma(x, y) \nu(y) d y .
$$

Remark 9. The fact the integral in (3.3) is well posed (finite) is a consequence of our result.

Remark 10. Theorem 6 can be read as that an $L$-superharmonic function $u$ (with no assumptions on its sign) can be decomposed into a positive superharmonic function $(w)$ and an harmonic function $(l)$. Clearly in general, this fact is not true without further assumptions. Indeed the function $u(x):=-|x|^{2}$ is superharmonic: $-\Delta u=2 N$, but it cannot be written as $u=l+w$ with $l$ harmonic and $w$ nonnegative and superharmonic ${ }^{2}$. In this respect, the hypothesis (3.2) plays a crucial role. Roughly speaking, the limit (3.2) or more explicitily (3.8), can be seen as a procedure to extract from a superharmonic function its harmonic component.

Notice that if we assume that the integral in (3.3) is finite everywhere, then this kind of decomposition is immediate. Hence we can claim that,

If the equation

$$
-L(h)=\nu \geq 0
$$

has a solution $u \in \mathscr{C}^{2}\left(\mathbb{R}^{N}\right)$ satisfying (3.2), then any solution $v$ of (3.9) can be decomposed into an harmonic function and a nonnegative superharmonic function.

Finally a natural question arises: is the ring condition (3.2) necessary to obtain such a decomposition? That is if $-L u=\nu \geq 0$ and assume that $u$ can be decomposed as above, i.e. $u(x)=h(x)+\int_{\mathbb{R}^{N}} \Gamma(x, y) \nu(y) d y$. Is the limit in (3.8) finite? A positive answer to this question is given by the claim $\mathrm{C}$ of Theorem 6 .

Remark 11. We emphasise that in Theorem 6-A we do not assume that $u$ is bounded from below. Indeed this fact is a consequence of the positivity of the fundamental solution and (3.6). In particular if (3.2) holds with $l \geq 0$, this gives a first answer to the positivity problem.

We note that a different representation formula under different assumptions (see in particular the assumptions on the symmetry of the matrix $A$ and on the not-totally degeneracy of $A$ ) has been proved in [1], under the hypothesis that $u$ is bounded from below. See Theorem 2.4.5 and Corollary 2.4.6 of [1] for details.

Remark 12. The ring condition (3.2) plays a central role in Theorem 6 , and the relation (3.5) give us a rate of convergence in the limit in (3.2), namely

$$
\int_{\Omega_{2 R}(x) \backslash \Omega_{R}(x)} \frac{\left(\nabla \Gamma_{x} \cdot A \nabla \Gamma_{x}\right)}{\Gamma_{x}}\left(u-l_{x}\right) d y \geq \frac{C}{R}, \quad \forall x \in \mathbb{R}^{N} \text { and for } R \text { large. }
$$

Therefore if the integral, for some $x \in \mathbb{R}^{N}$, vanishes too fast as $R \rightarrow+\infty$, then the function $u$ is harmonic.

\footnotetext{
${ }^{2}$ Indeed if this would be true, since $w$ is nonnegative it must satisfies $w(x) \geq C \int|x-y|^{2-n} 2 N d y=+\infty$.
} 
Furthermore the estimate (3.10), at least in this generality is sharp, in the sense that there exists a solution of $-L(u) \geq 0$ such that

$$
\limsup _{R \rightarrow+\infty} R \int_{\Omega_{2 R}(x) \backslash \Omega_{R}(x)} \frac{\left(\nabla \Gamma_{x} \cdot A \nabla \Gamma_{x}\right)}{\Gamma_{x}}\left(u-l_{x}\right) d y \leq C^{\prime}<+\infty .
$$

To this end consider $L=\Delta$ the Euclidean Laplacian operator, $N>2, u(z)=\left(1+|z|^{2}\right)^{(2-N) / 2}$ and $x=0$ and $l=0$. It is clear that $u$ is a strict positive superharmonic function. By computation, taking into account that

$$
A_{R}:=\Omega_{2 R}(0) \backslash \Omega_{R}(0)=\left\{(2 R)^{-1}<\left(|y|^{2-N}<R^{-1}\right\}=B_{(2 R)^{1 / N-2}} \backslash B_{R^{1 / N-2}},\right.
$$

we have

$$
\int_{A_{R}} \frac{\left(\nabla \Gamma_{0} \cdot A \nabla \Gamma_{0}\right)}{\Gamma_{0}} u d y=\int_{A_{R}} \frac{(N-2)^{2}}{|y|^{N}} \frac{1}{\left(1+|y|^{2}\right)^{(N-2) / 2}} d y \leq c R^{-\frac{N}{N-2}} R^{-1}\left|A_{R}\right|=c R^{-1},
$$

proving the sharpness of the rate $(3.10)$

The proof of Theorems 6 and 8 as well as some other sufficient conditions that guarantee the representation formulae (3.3) or (3.6) are contained in Lemma 26 and Lemma 29 below.

Remark 13. In the case $L=\Delta$, if $u$ is a nonnegative superharmonic function then from Theorem 6 it follows that $l$ is a positive classical harmonic function. Hence $l$ is a nonnegative constant by the classical Liouville theorem. In our general setting, the Liouville theorem may not be true. Actually, the next results claims the equivalence between Riesz representation result, Liouville type theorem and the validity of some ring condition as in (3.2).

Theorem 14. Assume that if $u \in L_{l o c}^{1}\left(\mathbb{R}^{N}\right)$ is a distributional solution of $L u=0$, then $u$ coincides almost everywhere with a $C^{2}\left(\mathbb{R}^{N}\right)$ function.

The following statements are equivalent.

1. Let $u \in \mathscr{C}^{2}\left(\mathbb{R}^{N}\right)$. Then $-L(u)=\nu \geq 0$ and $\inf u=m \in \mathbb{R}$ if and only if

$$
u(x)=m+\int_{\mathbb{R}^{N}} \Gamma(x, y) \nu(y) d y \quad \forall x \in \mathbb{R}^{N} .
$$

2. Let $u \in \mathscr{C}^{2}\left(\mathbb{R}^{N}\right)$. If $u$ is bounded from below and $-L(u)=0$ then $u$ is constant.

3. Let $u \in \mathscr{C}^{2}\left(\mathbb{R}^{N}\right)$ be such that $-L(u) \geq 0$ and $l \in \mathbb{R}$. Then, inf $u=l$ if and only if

$$
\liminf _{R \rightarrow+\infty} \int_{\Omega_{2 R}(x) \backslash \Omega_{R}(x)} \frac{\left(\nabla \Gamma_{x} \cdot A \nabla \Gamma_{x}\right)}{\Gamma_{x}}(u-l) d y=0 \quad \forall x \in \mathbb{R}^{N}
$$

if and only if

$$
\lim _{R \rightarrow+\infty} \int_{\Omega_{2 R}(x) \backslash \Omega_{R}(x)} \frac{\left(\nabla \Gamma_{x} \cdot A \nabla \Gamma_{x}\right)}{\Gamma_{x}}|u-l| d y=0 \quad \forall x \in \mathbb{R}^{N}
$$

if and only if

$$
\liminf _{R \rightarrow+\infty} \int_{\partial \Omega_{R}(x)} \frac{\left(\nabla \Gamma_{x} \cdot A \nabla \Gamma_{x}\right)}{\left|\nabla \Gamma_{x}\right|}(u-l) d H_{n-1}=0 \quad \forall x \in \mathbb{R}^{N}
$$


if and only if

$$
\lim _{R \rightarrow+\infty} \int_{\partial \Omega_{R}(x)} \frac{\left(\nabla \Gamma_{x} \cdot A \nabla \Gamma_{x}\right)}{\left|\nabla \Gamma_{x}\right|}|u-l| d H_{n-1}=0 \quad \forall x \in \mathbb{R}^{N}
$$

if and only if

$$
l=\lim _{R \rightarrow+\infty} \frac{1+\alpha}{R^{1+\alpha}} \int_{\Omega_{R}(x)} \frac{\left(\nabla \Gamma_{x} \cdot A \nabla \Gamma_{x}\right)}{\Gamma_{x}^{2+\alpha}} u d y \quad \alpha>-1 \forall x \in \mathbb{R}^{N} .
$$

4. Let $u \in \mathscr{C}^{2}\left(\mathbb{R}^{N}\right)$. Suppose that $u$ is bounded from below and $-L(u) \geq 0$. Set

$$
c_{x}:=\liminf _{R \rightarrow+\infty} \frac{1}{\ln 2} \int_{\Omega_{2 R}(x) \backslash \Omega_{R}(x)} \frac{\left(\nabla \Gamma_{x} \cdot A \nabla \Gamma_{x}\right)}{\Gamma_{x}} u d y \quad \forall x \in \mathbb{R}^{N},
$$

then $c_{x}$ does not depend on $x: c_{x}=c \in \mathbb{R}$.

If one of the above statements is true then $l=m=c$.

Remark 15. Condition 3. of the above Theorem 14, can be restated as

$$
\inf u=\liminf _{R \rightarrow+\infty} \frac{1}{\ln 2} \int_{\Omega_{2 R}(x) \backslash \Omega_{R}(x)} \frac{\left(\nabla \Gamma_{x} \cdot A \nabla \Gamma_{x}\right)}{\Gamma_{x}} u d y \quad \forall x \in \mathbb{R}^{N},
$$

provided the quantities involved are finite. A natural question arises: does the relation (3.18) hold even when the quantities are not finite?

If for some $x \in \mathbb{R}^{N}$

$$
\liminf _{R \rightarrow+\infty} \frac{1}{\ln 2} \int_{\Omega_{2 R}(x) \backslash \Omega_{R}(x)} \frac{\left(\nabla \Gamma_{x} \cdot A \nabla \Gamma_{x}\right)}{\Gamma_{x}} u d y=-\infty,
$$

then from (3.36) one deduces that $\inf u=-\infty$. The converse is not true as the following example shows. Let $L=\Delta$ the Laplacian in the Euclidean case, and let $u$ be a non constant harmonic function. Clearly $u$ is superharmonic and inf $u=-\infty$. However, since $u$ is harmonic, we have (see (3.44) below),

$\lim _{R \rightarrow+\infty} \frac{1}{\ln 2} \int_{\Omega_{2 R}(x) \backslash \Omega_{R}(x)} \frac{\left(\nabla \Gamma_{x} \cdot A \nabla \Gamma_{x}\right)}{\Gamma_{x}} u d y=\frac{1}{\ln 2} \int_{\Omega_{2 R}(x) \backslash \Omega_{R}(x)} \frac{\left(\nabla \Gamma_{x} \cdot A \nabla \Gamma_{x}\right)}{\Gamma_{x}} u d y=u(x) \in \mathbb{R}$.

Remark 16. An alternative formulation of Theorem 14 is the following. The classical Liouville theorem holds for the operator $L$ if and only if a representation formula for non negative supersolution holds. These properties are equivalent to the fact that the infimum of a supersolution can be characterised by the ring condition (3.12). Finally all the above statements are equivalent to the fact that the limits of the means involved in the conditions (3.12)-(3.17) on the ring centered at $x$ do not depend on $x$. Indeed they are constant.

Remark 17. The main Theorems 6 and 14 can be formulated even in a more general situation, for instance replacing the whole Euclidean space $\mathbb{R}^{N}$ with a domain $\Omega$ (open connected set).

To this end the hypotheses on the operator (and hence on the fundamental solution) must be changed accordingly replacing in all $H 1 \ldots H 7$., $\mathbb{R}^{N}$ with $\Omega$. In addition since $H 4$. involves a limit at infinity, we need to change it in the following way, 
$H 4$. for any $x \in \mathbb{R}^{N}$ and for any $z \in \partial \Omega, \lim _{y \rightarrow z} \Gamma(x, y)=0$ uniformly, that is

$$
\forall \epsilon>0, \exists n>0 \text { such that } \forall y \in \Omega \backslash C_{n}: \Gamma(x, y)<\epsilon
$$

where $C_{n}$ is the compact set $C_{n}:=\{z \in \Omega: \operatorname{dist}(z, \partial \Omega) \geq 1 / n$ and $|x| \leq n\}$.

With these changes Theorems 6 and 14 hold as they are stated replacing $\mathbb{R}^{N}$ with $\Omega$.

Very simple examples related to the Euclidean Laplacian are for instance, bounded regular domains for which there exists a Green function and half spaces.

Remark 18. In what follows we are going to show a variant of Theorem 14. For a given operator $L$ described in section 2. the classical Liouville theorem may not be true. However, it may be true for functions belonging to some subspace of $\mathscr{C}^{2}\left(\mathbb{R}^{N}\right)$. For instance for bounded functions or more generally for functions with a prescribed growth at infinity or even for a summability space. In this case the equivalences of the Theorem 14 remain valid.

Indeed we have,

Theorem 19. Let $X$ be a subspace of $\mathscr{C}^{2}\left(\mathbb{R}^{N}\right)$ satisfying ${ }^{3}$

$$
\text { if } u \in X \text { and } v \in \mathscr{C}^{2}\left(\mathbb{R}^{N}\right) \text { such that }|v| \leq u \text {, then } v \in X \text {. }
$$

If in Theorem 14 we replace the space $\mathscr{C}^{2}\left(\mathbb{R}^{N}\right)$ with $\mathscr{C}^{2}\left(\mathbb{R}^{N}\right) \cap X$, then the equivalences 1. $\Leftrightarrow 2 . . \Leftrightarrow 3 . \Leftrightarrow 4$. hold.

See subsections 3.1-3.5 for the proof.

Remark 20. In order to reformulate the ring condition (3.2) in particular cases we shall use the following notation. Fix $x \in \mathbb{R}^{N}$ and choose a real number $D_{x}>2$. Next we define $g_{x}(y):=\Gamma_{x}^{\frac{1}{2-D x}}(y)$. The function $g_{x}: \mathbb{R}^{N} \rightarrow \mathbb{R}$ is continuous, positive and smooth in $\mathbb{R}^{N} \backslash\{x\}$ and $g_{x}(x)=0$. We set $h_{x}^{2}:=\left(\nabla g_{x} \cdot A \nabla g_{x}\right)$. The function $g_{x}$ plays the the same role that the gauge $N_{2}\left(x^{-1} \circ \cdot\right)$ plays in the Carnot group setting. Notice that $y \in \Omega_{R}(x) \Leftrightarrow g_{x}(y)<R^{1 /\left(D_{x}-2\right)}$.

We wish to point out that the choice of $D_{x}>2$ is left free.

Let us to explicitly compute some relevant quantities involved in this paper. From definition of $g_{x}$ we have

$$
\frac{\left(\nabla \Gamma_{x} \cdot A \nabla \Gamma_{x}\right)}{\Gamma_{x}}=\left(2-D_{x}\right)^{2} g_{x}^{2-2 D_{x}} \frac{\left(\nabla g_{x} \cdot A \nabla g_{x}\right)}{g_{x}^{2-D_{x}}}=\left(2-D_{x}\right)^{2} \frac{h_{x}^{2}}{g_{x}^{D_{x}}}=\left(D_{x}-2\right)^{2} \Gamma^{\frac{D_{x}}{D_{x}-2}} h_{x}^{2} .
$$

Hence from Lemma 26 (see below) we see that

$$
\int_{\Omega_{2 R}(x) \backslash \Omega_{R}(x)} \Gamma^{\frac{D_{x}}{D_{x}-2}} h_{x}^{2} d y=\frac{1}{\left(2-D_{x}\right)^{2}} \int_{\Omega_{2 R}(x) \backslash \Omega_{R}(x)} \frac{\left(\nabla \Gamma_{x} \cdot A \nabla \Gamma_{x}\right)}{\Gamma_{x}} d y=\frac{\ln 2}{\left(2-D_{x}\right)^{2}},
$$

for any $R>0$ and $x \in \mathbb{R}^{N}$. This justifies why we shall refer to the integral

$$
\frac{1}{\ln 2} \int_{\Omega_{r}(x) \backslash \Omega_{r / 2}(x)} \frac{\left(\nabla \Gamma_{x} \cdot A \nabla \Gamma_{x}\right)}{\Gamma_{x}} u d y,
$$

\footnotetext{
${ }^{3}$ For instance $X=L^{p}\left(\mathbb{R}^{N}\right) \cap \mathscr{C}^{2}\left(\mathbb{R}^{N}\right)$ or if $\left.\left.G: \mathbb{R}^{N} \rightarrow\right] 0,+\infty\right]$ is a positive function then $X$ could be the space of functions with a growth prescribed by the weight $G$, namely

$$
X:=\left\{u \in \mathscr{C}^{2}\left(\mathbb{R}^{N}\right): \exists c>0,|u(x)| \leq c G(x) \forall x \in \mathbb{R}^{N}\right\} .
$$
}


as a mean.

With this notation the ring conditions (3.2), see also Lemma 26, can be stated as

$$
\liminf _{R \rightarrow+\infty} \int_{R<g_{x}(y)<2 R} \frac{h_{x}^{2}(y)}{g_{x}^{D_{x}(y)}}\left(u(y)-l_{x}\right) d y=0 .
$$

For instance in the case $L$ is a sub-Laplacian on a Carnot group, $D_{x}=Q>2$ is constant and $g_{x}(y)=N_{2}\left(x^{-1} \circ y\right)$ where $N_{2}$ is a symmetric homogeneous norm. With this notation, $h_{x}^{2}=\psi_{x}^{2}$ and it is bounded, $\Omega_{r}(x)=\left\{y \in \mathbb{R}^{N} \mid N_{2}(x, y)<r^{\frac{1}{Q-2}}\right\}$ and since $\left|\Omega_{r}(x)\right|=\left|B_{N}\left(r^{\frac{1}{Q-2}}\right)\right|=C r^{\frac{Q}{Q-2}}$, for functions that are bounded from below the condition (3.21) and hence (3.2), becomes

$$
\liminf _{R \rightarrow+\infty} f_{R<N_{2}\left(x^{-1} \circ y\right)<2 R}\left|\nabla_{L} N_{2}\left(x^{-1} \circ y\right)\right|^{2}(u(y)-l) d y=0,
$$

where $f_{U}:=\frac{1}{|U|} \int_{U}$

See subsection 4 for the analysis in Carnot Groups.

Moreover in the special case in which the Carnot group is the classical Euclidean space with $L=\Delta$, then (3.2) for functions that are bounded from below can be read as follows,

$$
\liminf _{R \rightarrow+\infty} f_{R<|x-y|<2 R}(u(y)-l) d y=0 .
$$

\subsection{Lemmata}

In this subsection we prove some lemmata which will be used in proving Theorems 6,8 and 14.

In similar settings in $[15,7,8]$ the authors proved the implication $3 . \Rightarrow 1$. of Theorem 14 directly using a technique based on the test functions method. Even in our setting this technique can be applied: in this case we have to choose tests functions that depends on the fundamental solution of $L$. This idea is used in subsections 3.2 and 3.6.1. However in this paper for the proof of the representation formulae we shall use a different pattern. Indeed we will give a proof which is based on the local representation formulae (3.25) and (3.26). This choice is motivated by the fact that even the other implications are based on the local representation formula for a function $u$.

As a consequence of divergence theorem, we have that

$$
\int_{U} L u=\int_{\partial U}(A \nabla u \cdot \nu) d H^{N-1}
$$

for any smooth open set $U$ and any $u$ smooth function on $\bar{U}$. In (3.24), $\nu$ denotes the exterior normal unitary vector to $\partial U$ and $d H^{N-1}$ denotes the the $(N-1)$-dimensional Hausdorff measure.

We shall use (3.24) with $U=\Omega_{r}(x)$. We notice that by Sard's Lemma these sets are regular for almost every $r>0$.

Arguing as in [9], we have the following. 
Lemma 21. Let $u \in \mathscr{C}^{2}\left(\mathbb{R}^{N}\right), x \in \mathbb{R}^{N}, R>0,0<\delta<1, \alpha \neq-1$, for a.e. $r>0$, then

$$
\begin{aligned}
& u(x)= \int_{\partial \Omega_{r}(x)} \frac{\left(\nabla \Gamma_{x} \cdot A \nabla \Gamma_{x}\right)}{\left|\nabla \Gamma_{x}\right|} u d H_{n-1}+\int_{\Omega_{r}(x)}(-L u)\left(\Gamma_{x}-\frac{1}{r}\right) d y \\
& u(x)= \frac{1}{-\ln \delta}\left\{\int_{\Omega_{R}(x) \backslash \Omega_{\delta R}(x)} \frac{\left(\nabla \Gamma_{x} \cdot A \nabla \Gamma_{x}\right)}{\Gamma_{x}} u d y+\right. \\
&\left.\quad+\int_{\delta R}^{R} \frac{1}{r} \int_{\Omega_{r}(x)}(-L u)\left(\Gamma_{x}-\frac{1}{r}\right) d y d r\right\}, \\
& u(x)=\frac{\alpha+1}{\left(1-\delta^{\alpha+1}\right) R^{\alpha+1}}\left\{\int_{\Omega_{R}(x) \backslash \Omega_{\delta R}(x)} \frac{\left(\nabla \Gamma_{x} \cdot A \nabla \Gamma_{x}\right)}{\Gamma_{x}^{\alpha+2}} u d y+\right. \\
&\left.\quad+\int_{\delta R}^{R} r^{\alpha} \int_{\Omega_{r}(x)}(-L u)\left(\Gamma_{x}-\frac{1}{r}\right) d y d r\right\},
\end{aligned}
$$

and $\delta=0$ is allowed in (3.27) provided $\alpha>-1$.

Moreover if $\left|A^{\prime} \nabla \Gamma\right| \in L_{l o c}^{1}\left(\mathbb{R}^{N}\right)$, then

$$
u(x)=\int_{\partial \Omega_{r}(x)} \frac{\left(\nabla \Gamma_{x} \cdot A \nabla \Gamma_{x}\right)}{\left|\nabla \Gamma_{x}\right|} u d H_{n-1}+\int_{\Omega_{r}(x)}\left(\nabla \Gamma_{x} \cdot A \nabla u\right) d y .
$$

Proof. In what follows the dependence on $x$ will be omitted, hence $\Omega_{s}$ stands for $\Omega_{s}(x)$ and $\Gamma$ stands for $\Gamma_{x}$. Let $0<\epsilon<r$ and set $U_{\epsilon}:=\Omega_{r} \backslash \overline{\Omega_{\epsilon}}$. Since $-L^{*} \Gamma=0$ on $U_{\epsilon}$, we have

$$
\begin{gathered}
\int_{U_{\epsilon}}\left(A^{\prime} \nabla \Gamma \cdot \nabla u\right)=\int_{\partial U_{\epsilon}}\left(A^{\prime} \nabla \Gamma \cdot \nu\right) u d H^{N-1}-\int_{U_{\epsilon}}\left(L^{*} \Gamma\right) u \\
=\int_{\partial \Omega_{r}}\left(A^{\prime} \nabla \Gamma \cdot \nu\right) u d H^{N-1}-\int_{\partial \Omega_{\epsilon}}\left(A^{\prime} \nabla \Gamma \cdot \nu\right) u d H^{N-1} .
\end{gathered}
$$

On the other hand,

$$
\begin{array}{r}
\int_{U_{\epsilon}}\left(A^{\prime} \nabla \Gamma \cdot \nabla u\right) d y=\int_{\frac{1}{r}<\Gamma<\frac{1}{\epsilon}}\left(\nabla\left(\Gamma-\frac{1}{r}\right) \cdot A \nabla u\right) d y \\
=\int_{\partial \Omega_{r}}\left(\Gamma-\frac{1}{r}\right)(A \nabla u \cdot \nu) d H^{N-1}-\int_{\partial \Omega_{\epsilon}}\left(\Gamma-\frac{1}{r}\right)(A \nabla u \cdot \nu) d H^{N-1}-\int_{\frac{1}{r}<\Gamma<\frac{1}{\epsilon}}\left(\Gamma-\frac{1}{r}\right) L u d y \\
=-\left(\frac{1}{\epsilon}-\frac{1}{r}\right) \int_{\partial \Omega_{\epsilon}}(A \nabla u \cdot \nu) d H^{N-1}-\int_{\frac{1}{r}<\Gamma<\frac{1}{\epsilon}}\left(\Gamma-\frac{1}{r}\right) L u d y \\
=-\left(\frac{1}{\epsilon}-\frac{1}{r}\right) \int_{\Omega_{\epsilon}} L u d y-\int_{\frac{1}{r}<\Gamma<\frac{1}{\epsilon}}\left(\Gamma-\frac{1}{r}\right) L u d y,
\end{array}
$$

where in the last identity we have used (3.24).

Let $\phi \in \mathscr{C}_{0}^{\infty}\left(\mathbb{R}^{N}\right)$ such that $\phi=1$ on $\overline{\Omega_{\epsilon}}$. By hypotheses we have

$$
u(x)=-\int_{\mathbb{R}^{N}} \Gamma_{x} L(u \phi) d y=-\lim _{\epsilon \rightarrow 0} \int_{\Gamma \leq \frac{1}{\epsilon}} \Gamma_{x} L(u \phi) d y .
$$

Therefore integrating by parts, and taking into account that $\phi(y)=1$ for $\Gamma_{x}(y)=\frac{1}{\epsilon}$, we obtain

$$
\begin{gathered}
-\int_{\Gamma \leq \frac{1}{\epsilon}} \Gamma_{x} L(u \phi) d y=\int_{\Gamma \leq \frac{1}{\epsilon}}\left(A^{\prime} \nabla \Gamma_{x} \cdot \nabla(u \phi)\right) d y-\frac{1}{\epsilon} \int_{\partial \Omega_{\epsilon}}(A \nabla(u \phi) \cdot \nu) d H^{N-1} \\
=-\int_{\Gamma \leq \frac{1}{\epsilon}} L^{*}\left(\Gamma_{x}\right)(u \phi) d y-\int_{\partial \Omega_{\epsilon}}\left(A^{\prime} \nabla \Gamma_{x} \cdot \nu\right) u \phi d H^{N-1}-\frac{1}{\epsilon} \int_{\partial \Omega_{\epsilon}}(A \nabla u \cdot \nu) d H^{N-1} \\
=-\int_{\partial \Omega_{\epsilon}}\left(A^{\prime} \nabla \Gamma_{x} \cdot \nu\right) u d H^{N-1}-\frac{1}{\epsilon} \int_{\Omega_{\epsilon}} L u d y
\end{gathered}
$$


where in the last identity we have used the fact that $L^{*}\left(\Gamma_{x}(y)\right)=0$ for $y \neq x$ and the relation (3.24). Gluing together the last relations, and observing that the normal $\nu$ can be written as $\nu=-\frac{\nabla \Gamma}{|\nabla \Gamma|}$ we have

$$
u(x)=\int_{\partial \Omega_{r}}\left(A^{\prime} \nabla \Gamma_{x} \cdot \frac{\nabla \Gamma_{x}}{\left|\nabla \Gamma_{x}\right|}\right) u d H^{N-1}-\int_{\Omega_{r}}\left(\Gamma-\frac{1}{r}\right) L u d y+\lim _{\epsilon \rightarrow 0}\left(\frac{1}{r}-\frac{2}{\epsilon}\right) \int_{\Omega_{\epsilon}} L u d y .
$$

From (2.2) we get (3.25).

The identity (3.28) follows formally from (3.25) by integration by parts. In a more precise way, as before gluing (3.30), (3.29) and (3.31), we have

$$
u(x)=\lim _{\epsilon \rightarrow 0} \int_{U_{\epsilon}}\left(A^{\prime} \nabla \Gamma \cdot \nabla u\right) d y-\int_{\partial \Omega_{r}}\left(A^{\prime} \nabla \Gamma \cdot \nu\right) u d H^{N-1}-\frac{1}{\epsilon} \int_{\Omega_{\epsilon}} L u d y
$$

which yields (3.28).

In order to obtain the missing relations, it is enough to multiply (3.25) by $r^{\alpha}$, then integrate with respect to variable $r$ between $[\delta R, R]$ and using the coarea formula to show that

$$
\begin{aligned}
\int_{\delta R}^{R}\left(r^{\alpha} \int_{\partial \Omega_{r}} \frac{\left(\nabla \Gamma_{x} \cdot A \nabla \Gamma_{x}\right)}{\left|\nabla \Gamma_{x}\right|} u\right) d r & =\int_{\delta R}^{R} \int_{\partial \Omega_{r}} \frac{\left(\nabla \Gamma_{x} \cdot A \nabla \Gamma_{x}\right)}{\Gamma^{\alpha}\left|\nabla \Gamma_{x}\right|} u d r \\
& =\int_{\Omega_{R}(x) \backslash \Omega_{\delta R}(x)} \frac{\left(\nabla \Gamma_{x} \cdot A \nabla \Gamma_{x}\right)}{\Gamma_{x}^{\alpha+2}} u d y
\end{aligned}
$$

This completes the proof of (3.26) and (3.27) for $\delta>0$.

In order to get the proof for $\delta=0$, we remark that choosing $u=1$ in (3.27) we have

$$
1=\frac{\alpha+1}{\left(1-\delta^{\alpha+1}\right) R^{\alpha+1}} \int_{\Omega_{R}(x) \backslash \Omega_{\delta R}(x)} \frac{\left(\nabla \Gamma_{x} \cdot A \nabla \Gamma_{x}\right)}{\Gamma_{x}^{\alpha+2}} d y .
$$

By letting $\delta \rightarrow 0$ we have that $\frac{\left(\nabla \Gamma_{x} \cdot A \nabla \Gamma_{x}\right)}{\Gamma_{x}^{\alpha+2}} \in L_{l o c}^{1}\left(\mathbb{R}^{N}\right)$, and hence we can pass to the limit (as $\delta \rightarrow 0$ ) in (3.27) completing the proof.

Remark 22. With the notation of Remark 20, by a rescaling $\left(r \rightarrow r^{D_{x}-1}\right)$, the identities (3.25), (3.26) and (3.27) of Lemma 21, for any $x \in \mathbb{R}^{N}, R>0,0<\delta<1, \beta \neq 0$, and for a.e. $r>0$, read respectively as

$$
\begin{aligned}
& u(x)=\frac{D_{x}-2}{r^{D_{x}-1}} \int_{g_{x}=r} \frac{h_{x}^{2}}{\left|\nabla g_{x}\right|} u d H_{n-1}+\int_{g_{x}<r}(-L u)\left(\Gamma_{x}-\frac{1}{r^{D_{x}-2}}\right) d y \\
& u(x)=\frac{1}{-\ln \delta}\left\{\left(D_{x}-2\right) \int_{\delta R<g_{x}<R} \frac{h_{x}^{2}}{g_{x}^{D_{x}}}(y) u(y) d y+\right. \\
& \left.+\int_{\delta R}^{R} \frac{1}{r} \int_{g_{x}<r}(-L u)\left(\Gamma_{x}-\frac{1}{r^{D_{x}-2}}\right) d y d r\right\}, \\
& u(x)=\frac{\beta}{\left(1-\delta^{\beta}\right) R^{\beta}}\left\{\left(D_{x}-2\right) \int_{\delta R<g_{x}<R} \frac{h_{x}^{2}}{g_{x}^{D_{x}-\beta}} u d y\right. \\
& \left.+\int_{\delta R}^{R} \frac{r^{\beta}}{r} \int_{g_{x}<r}(-L u)\left(\Gamma_{x}-\frac{1}{r^{D_{x}-2}}\right) d y d r\right\},
\end{aligned}
$$

and $\delta=0$ is allowed in (3.34) provided $\beta>0$. 
Choosing $u \equiv 1$ in Lemma 21 we have

Corollary 23. Let $x \in \mathbb{R}^{N}, \alpha>-1$, then

$$
\begin{aligned}
& \int_{\partial \Omega_{r}(x)} \frac{\left(\nabla \Gamma_{x} \cdot A \nabla \Gamma_{x}\right)}{\left|\nabla \Gamma_{x}\right|} d H_{n-1}=1 \text { for a.e. } r>0 ; \\
& \frac{1}{\ln \eta} \int_{\Omega_{\eta R}(x) \backslash \Omega_{R}(x)} \frac{\left(\nabla \Gamma_{x} \cdot A \nabla \Gamma_{x}\right)}{\Gamma_{x}} d y=1 \quad \forall R>0, \eta>1 ; \\
& \frac{\alpha+1}{R^{\alpha+1}} \int_{\Omega_{R}(x)} \frac{\left(\nabla \Gamma_{x} \cdot A \nabla \Gamma_{x}\right)}{\Gamma_{x}^{\alpha+2}} d y=1 \quad \forall R>0 .
\end{aligned}
$$

Lemma 24. Let $u \in \mathscr{C}^{2}\left(\mathbb{R}^{N}\right)$ be such that $-L(u) \geq 0$. For any $x \in \mathbb{R}^{N}$, the functions

$$
\begin{aligned}
r \longmapsto & N_{x}(r):=\int_{\partial \Omega_{r}(x)} \frac{\left(\nabla \Gamma_{x} \cdot A \nabla \Gamma_{x}\right)}{\left|\nabla \Gamma_{x}\right|} u d H_{n-1}, \\
r & \longmapsto M_{x}(r):=\int_{\Omega_{r}(x)}(-L u)\left(\Gamma_{x}-\frac{1}{r}\right) d y,
\end{aligned}
$$

are nonnincreasing and nondecreasing, respectively.

Moreover for any $x \in \mathbb{R}^{N}$ and $R>0$, there holds

$$
\begin{aligned}
& u(x) \geq \int_{\partial \Omega_{r}(x)} \frac{\left(\nabla \Gamma_{x} \cdot A \nabla \Gamma_{x}\right)}{\left|\nabla \Gamma_{x}\right|} u d H_{n-1}, \\
& u(x) \geq \frac{1}{\ln \eta} \int_{\Omega_{\eta R}(x) \backslash \Omega_{R}(x)} \frac{\left(\nabla \Gamma_{x} \cdot A \nabla \Gamma_{x}\right)}{\Gamma_{x}} u d y, \quad \eta>1 \\
& \left.u(x) \geq \frac{\alpha+1}{\left(1-\delta^{\alpha+1}\right) R^{\alpha+1}} \int_{\Omega_{R}(x) \backslash \Omega_{\delta R}(x)} \frac{\left(\nabla \Gamma_{x} \cdot A \nabla \Gamma_{x}\right)}{\Gamma_{x}^{\alpha+2}} u d y, \quad R>0, \alpha>-1,1>\delta \geq(B) .42\right)
\end{aligned}
$$

In particular if $L(u)=0$ then

$$
\begin{aligned}
u(x) & =\int_{\partial \Omega_{r}(x)} \frac{\left(\nabla \Gamma_{x} \cdot A \nabla \Gamma_{x}\right)}{\left|\nabla \Gamma_{x}\right|} u d H_{n-1}, \\
u(x) & =\frac{1}{\ln \eta} \int_{\Omega_{\eta R}(x) \backslash \Omega_{R}(x)} \frac{\left(\nabla \Gamma_{x} \cdot A \nabla \Gamma_{x}\right)}{\Gamma_{x}} u d y, \quad \eta>1 .
\end{aligned}
$$

Proof. Let $s>r$. Since the integrand in $M_{x}$ is nonnegative and $\Omega_{r} \subset \Omega_{s}$, we have

$$
M_{x}(s)=\int_{\Omega_{s}(x)}(-L u)\left(\Gamma_{x}-\frac{1}{s}\right) d y \geq \int_{\Omega_{r}(x)}(-L u)\left(\Gamma_{x}-\frac{1}{s}\right) d y \geq M_{x}(r) .
$$

On the other hand from $(3.25), M_{x}(r)+N_{x}(r)=u(x)$ is constant with respect to $r$. Thus $N_{x}$ is non-increasing.

The remaining relations are an easy consequence of the identities in Lemma 21 and the fact that $-L(u) \geq 0$.

Lemma 25. Let $\nu$ be a regular measure. Let $w \in L_{l o c}^{1}\left(\mathbb{R}^{N}\right)$ defined by

$$
w(x):=\int_{\mathbb{R}^{N}} \Gamma(x, y) \nu(y) d y \quad \text { a.e } x \in \mathbb{R}^{N} .
$$

Then $-L(w)=\nu$ in the distributional sense. 
Proof. To this end it is enough to show that, for any $\phi \in \mathscr{C}_{0}^{\infty}\left(\mathbb{R}^{N}\right)$ we have,

$$
\int_{\mathbb{R}^{N}}-L^{*}(\phi)(x) w(x)=\int_{\mathbb{R}^{N}} \phi(x) \nu(x) .
$$

Indeed, multiplying $w$ by $-L^{*}(\phi)$ we have,

$$
\int_{\mathbb{R}^{N}}-L^{*}(\phi)(x) w(x) d x=\int_{\mathbb{R}^{N}} \int_{\mathbb{R}^{N}}-L^{*}(\phi)(x) \Gamma(x, y) \nu(y) d y d x=\int_{\mathbb{R}^{N}} \phi(y) \nu(y) .
$$

Here we have used the fact that $\Gamma(\cdot, y)$ is a fundamental solution of $-L^{*}$.

Lemma 26. Let $u \in \mathscr{C}^{2}\left(\mathbb{R}^{N}\right)$ be such that $-L(u) \geq 0$. Let $x \in \mathbb{R}^{N}$ and $l_{x} \in \mathbb{R}$. Then the following implications hold.

$$
\begin{aligned}
& (3.47) \Rightarrow(3.46) \Rightarrow(3.51) \Leftrightarrow(3.50) \Leftrightarrow(3.54) \\
& \Downarrow \quad \Uparrow \quad \mathbb{} \quad \mathbb{} \\
& (3.49) \Rightarrow(3.48) \Rightarrow(3.53) \Leftrightarrow(3.52)
\end{aligned}
$$

where, for $\eta>1$,

$$
\begin{array}{r}
\liminf _{R \rightarrow+\infty} \int_{\partial \Omega_{R}(x)} \frac{\left(\nabla \Gamma_{x} \cdot A \nabla \Gamma_{x}\right)}{\left|\nabla \Gamma_{x}\right|}\left|u-l_{x}\right| d H_{n-1}=0 ; \\
\lim _{R \rightarrow+\infty} \int_{\partial \Omega_{R}(x)} \frac{\left(\nabla \Gamma_{x} \cdot A \nabla \Gamma_{x}\right)}{\left|\nabla \Gamma_{x}\right|}\left|u-l_{x}\right| d H_{n-1}=0 ; \\
\liminf _{R \rightarrow+\infty} \int_{\Omega_{\eta R}(x) \backslash \Omega_{R}(x)} \frac{\left(\nabla \Gamma_{x} \cdot A \nabla \Gamma_{x}\right)}{\Gamma_{x}}\left|u-l_{x}\right| d y=0 ; \\
\lim _{R \rightarrow+\infty} \int_{\Omega_{\eta R}(x) \backslash \Omega_{R}(x)} \frac{\left(\nabla \Gamma_{x} \cdot A \nabla \Gamma_{x}\right)}{\Gamma_{x}}\left|u-l_{x}\right| d y=0 ; \\
\liminf _{R \rightarrow+\infty} \int_{\partial \Omega_{R}(x)} \frac{\left(\nabla \Gamma_{x} \cdot A \nabla \Gamma_{x}\right)}{\left|\nabla \Gamma_{x}\right|} u d H_{n-1}=l_{x} ; \\
\liminf _{R \rightarrow+\infty} \frac{1}{\ln \eta} \int_{\Omega_{\eta R}(x) \backslash \Omega_{R}(x)} \frac{\left(\nabla \Gamma_{x} \cdot A \nabla \Gamma_{x}\right)}{\left|\nabla \Gamma_{x}\right|} u d H_{n-1}=l_{x} ; \\
\lim _{R \rightarrow+\infty} \frac{1}{\ln \eta} \int_{\Omega_{\eta R}(x) \backslash \Omega_{R}(x)} \frac{\left(\nabla \Gamma_{x} \cdot A \nabla \Gamma_{x}\right)}{\Gamma_{x}} u d y=l_{x} ; \\
\lim _{R \rightarrow+\infty} \frac{1+\alpha}{R^{1+\alpha}} \int_{\Omega_{R}(x)} \frac{\left(\nabla \Gamma_{x} \cdot A \nabla \Gamma_{x}\right)}{\Gamma_{x}^{2+\alpha}} u d y=l_{x} \quad \alpha>-1 .
\end{array}
$$

Moreover, if one of the above statements holds, then

$$
u(x)=l_{x}+\int_{\mathbb{R}^{N}} \Gamma(x, y) \nu(y) d y .
$$

Here $\nu:=-L(u)$. If (3.52), or equivalently (3.53) or (3.50) or (3.51), holds and $u(y) \geq l_{x}$ for any $y \in \mathbb{R}^{N}$, then all the above statements are equivalent. 
Proof. Taking into account Corollary 23 , the implications $(3.47) \Rightarrow(3.46),(3.47) \Rightarrow(3.51) \Rightarrow$ (3.50), (3.49) $\Rightarrow(3.48),(3.49) \Rightarrow(3.53) \Rightarrow(3.52)$, are obvious.

Without loss of generality we can assume $l_{x}=0$. Let us also remind that $N_{x}(r)$ is non increasing with respect to $r$ as stated in Lemma 24. We will use the following notation

$$
\begin{aligned}
K_{x} & :=\frac{\left(\nabla \Gamma_{x} \cdot A \nabla \Gamma_{x}\right)}{\left|\nabla \Gamma_{x}\right|}, \\
\mathcal{K}_{x} & :=\frac{\left(\nabla \Gamma_{x} \cdot A \nabla \Gamma_{x}\right)}{\Gamma_{x}} .
\end{aligned}
$$

$(3.46) \Rightarrow(3.51)$. Since $\liminf _{R(x) \rightarrow \infty} \int_{\partial \Omega_{R(x)}} K_{x}|u| d \sigma=0$, there exists a sequence $\left(R_{n}\right)$ such that $\lim _{n} \int_{\partial \Omega_{R_{n}}(x)} K_{x}|u| d \sigma=0$. Therefore $\lim _{n} \int_{\partial \Omega_{R_{n}(x)}} K_{x}|u| d \sigma=0$, that is $N_{x}\left(R_{n}\right) \rightarrow 0$. From the monotonicity of $N_{x}$ we get the claim.

$(3.50) \Rightarrow(3.51)$. It follows from monotonicity of $N_{x}$.

$(3.48) \Rightarrow(3.46)$. Arguing by contradiction, we suppose that

$$
\liminf _{r} \int_{\partial \Omega_{r}(x)} K_{x}|u| d \sigma=c>0
$$

This implies that for $r$ large, we have

$$
\int_{\partial \Omega_{r}(x)} K_{x}|u| d \sigma \geq c / 2
$$

Using the coarea formula we have

$$
\int_{\Omega_{\eta R}(x) \backslash \Omega_{R}(x)} \mathcal{K}_{x}|u| d y=\int_{R}^{\eta R} \frac{1}{r} \int_{\partial \Omega_{r}} K_{x}|u| d \sigma \geq \frac{c}{2} \int_{R}^{\eta R} \frac{d r}{r}=\frac{c \ln \eta}{2}>0,
$$

which contradicts the assumption (3.48).

$(3.47) \Rightarrow(3.49)$ Let $\epsilon>0$. By assumption (3.47) there exist $r_{0}>0$ such that for $r>r_{0}$ we have $\int_{\partial \Omega_{r}(x)} K_{x}|u|<\epsilon$. Integrating we have

$$
\int_{\Omega_{\eta R}(x) \backslash \Omega_{R}(x)} \mathcal{K}_{x}|u| d y=\int_{R}^{\eta R} \frac{1}{r} \int_{\partial \Omega_{r}} K_{x}|u|<\epsilon \int_{R}^{\eta R} \frac{d r}{r}=\epsilon \ln \eta .
$$

$(3.51) \Rightarrow(3.53)$ Since $N_{x}$ is nonincreasing we have

$$
\begin{aligned}
\ln \eta N_{x}(\eta R) & =\int_{R}^{\eta R} N_{x}(\eta R) \frac{d r}{r} \leq \int_{R}^{\eta R} N_{x}(r) \frac{d r}{r}=\int_{\Omega_{\eta R}(x) \backslash \Omega_{R}(x)} \mathcal{K}_{x} u d y \\
\ln \eta N_{x}(R) & =\int_{R}^{\eta R} N_{x}(R) \frac{d r}{r} \geq \int_{R}^{\eta R} N_{x}(r) \frac{d r}{r}=\int_{\Omega_{\eta R}(x) \backslash \Omega_{R}(x)} \mathcal{K}_{x} u d y .
\end{aligned}
$$

Since $N(R) \rightarrow 0$ (as $R \rightarrow \infty)$, these inequalities imply the claim.

$(3.52) \Rightarrow(3.50)$ Let

$$
b:=\liminf _{r \rightarrow \infty} N_{x}(r) .
$$

Arguing as in the proof of the implication $(3.51) \Rightarrow(3.53)$ we have that

$$
\liminf _{R \rightarrow \infty} \frac{1}{\ln \eta} \int_{\Omega_{\eta R}(x) \backslash \Omega_{R}(x)} \mathcal{K}_{x} u d y=b .
$$


This implies $b=0$.

$(3.51) \Rightarrow(3.54)$ Since $N_{x}$ is nonincreasing we have,

$$
\frac{R^{1+\alpha}}{1+\alpha} N_{x}(R)=\int_{0}^{R} N_{x}(R) r^{\alpha} d r \leq \int_{0}^{R} N_{x}(r) r^{\alpha} d r=\int_{\Omega(R)} \frac{\mathcal{K}_{x}}{\Gamma_{x}^{1+\alpha}} u d y .
$$

Hence,

$$
N_{x}(R) \leq \frac{1+\alpha}{R^{1+\alpha}} \int_{\Omega(R)} \frac{\mathcal{K}_{x}}{\Gamma_{x}^{1+\alpha}} u,
$$

which implies

$$
0 \leq \liminf \frac{1+\alpha}{R^{1+\alpha}} \int_{\Omega(R)} \frac{\mathcal{K}_{x}}{\Gamma_{x}^{1+\alpha}} u d y
$$

The reverse inequality follows from the simple calculation,

$$
\begin{gathered}
\frac{1+\alpha}{R^{1+\alpha}} \int_{\Omega(R)} \frac{\mathcal{K}_{x}}{\Gamma_{x}^{1+\alpha}} u d r=\frac{1+\alpha}{R^{1+\alpha}} \int_{0}^{R} r^{\alpha} N_{x}(r) d r= \\
\frac{1+\alpha}{R^{1+\alpha}}\left(\int_{0}^{s} r^{\alpha} N_{x}(r) d r+\int_{s}^{R} r^{\alpha} N_{x}(r) d r\right) \leq \frac{1+\alpha}{R^{1+\alpha}} \int_{0}^{s} r^{\alpha} N_{x}(r) d r+\frac{1+\alpha}{R^{1+\alpha}} N_{x}(s) \int_{s}^{R} r^{\alpha} d r= \\
\frac{1+\alpha}{R^{1+\alpha}} \int_{0}^{s} r^{\alpha} N_{x}(r) d r+N_{x}(s) \frac{R^{1+\alpha}-s^{1+\alpha}}{R^{1+\alpha}}
\end{gathered}
$$

where $0<s<R$. Indeed, letting $R \rightarrow+\infty$ we get,

$$
0 \leq \underset{R}{\limsup } \frac{1+\alpha}{R^{1+\alpha}} \int_{\Omega(R)} \frac{\mathcal{K}_{x}}{\Gamma_{x}^{1+\alpha}} u d y \leq N_{x}(s),
$$

which implies the claim.

$(3.54) \Rightarrow(3.51)$ Let $b:=\liminf _{r \rightarrow \infty} N_{x}(r)=\lim _{r \rightarrow \infty} N_{x}(r)$. We note that $b=-\infty$ is allowed. From (3.55) we get $b \leq 0$. Taking the limit for $R \rightarrow+\infty$ in (3.1) we obtain $N_{x}(s) \geq 0$ which implies the claim.

To conclude the proof assume that (3.51) is satisfied and $u(y) \geq l_{x}$ for any $y \in \mathbb{R}^{N}$. Therefore $\left|u(y)-l_{x}\right|=u(y)-l_{x}$ then (3.47) holds. Thus from the scheme (3.45) it follows that all implications are verified.

Remark 27. The missing implication $(3.53) \Longrightarrow(3.48)$ in general is not true. Indeed, in the Euclidean setting with $L=\Delta$, we choose $u(x)=u\left(x_{1}, \ldots, x_{N}\right):=x_{1}$. This function is harmonic in $\mathbb{R}^{N}$. Hence by (3.26) and using the notation of the above proof, we have

$$
u(0)=0=\frac{1}{\ln 2} \int_{\Omega_{2 R}(x) \backslash \Omega_{R}(x)} \mathcal{K}_{x} u d y .
$$

However

$$
\frac{1}{\ln 2} \int_{\Omega_{2 R}(x) \backslash \Omega_{R}(x)} \mathcal{K}_{x}|u| d y=C R \rightarrow+\infty
$$




\subsection{A priori estimates and the ring condition}

In this section we do not make any assumption on the sign of the solution $u$. We begin with an universal estimate which involves a ring condition.

Let $q>1$, in what follows by $q$-admissible function we mean a function $\phi \in \mathscr{C}_{0}^{\infty}(\mathbb{R})$ in the form $\phi=\varphi_{0}^{\gamma}$ with $\gamma>\frac{2 q}{q-1}$ an integer and $\varphi_{0} \in \mathscr{C}_{0}^{\infty}(\mathbb{R})$ such that $0 \leq \varphi_{0} \leq 1, \varphi_{0}(t)=1$ if $|t| \leq 1, \varphi_{0}(t)=0$ if $|t| \geq 2$.

Theorem 28. Let $q>1$. For any $\phi_{1} \in \mathscr{C}_{0}^{\infty}(\mathbb{R}) q$-admissible function, there exists $M$ such that if $u$ is a weak solution of $-L(u) \geq \nu \geq 0$, then we have

$$
\int_{\Omega_{2 R}(x)} \phi_{R}(y) \nu(y) d y \leq \frac{M}{R^{2}} \int_{\Omega_{2 R}(x) \backslash \Omega_{R}(x)}|u(y)| \phi_{R}^{1 / q}(y) \frac{\left(\nabla \Gamma_{x}(y) \cdot A(y) \nabla \Gamma_{x}(y)\right)}{\Gamma_{x}^{4}(y)} d y
$$

for any $R>0$ and a.e. $x \in \mathbb{R}^{N}$, where $\phi_{R}(y):=\phi_{1}\left(\frac{1}{R \Gamma_{x}(y)}\right)$.

In particular we have

$$
\int_{\Omega_{2 R}(x)} \phi_{R}(y) \nu(y) d y \leq 8 M R \int_{\Omega_{2 R}(x) \backslash \Omega_{R}(x)}|u(y)| \phi_{R}^{1 / q}(y) \frac{\left(\nabla \Gamma_{x}(y) \cdot A(y) \nabla \Gamma_{x}(y)\right)}{\Gamma_{x}(y)} d y .
$$

Here the constant $M$ depends only on $\phi_{1}: M:=\sup _{1<t<2} \frac{\left|\phi_{1}^{\prime \prime}(t)+2 \phi_{1}^{\prime}(t) / t\right|}{\phi_{1}^{1 / q}(t)}<+\infty$.

Proof. In what follows we shall omit to write the dependence on $x\left(\Omega_{R}=\Omega_{R}(x), \Gamma=\Gamma_{x}\right)$.

From our choice of $\phi_{R}$ it follows that it vanishes outside $\Omega_{2 R}(x)$ and $\phi_{R}=1$ in $\Omega_{R}(x)$. Using $\phi_{R}$ in Definition 3.1 we have

$$
\int \phi_{R} \nu \leq-\int u L^{*}\left(\phi_{R}\right) \leq \int|u|\left|\operatorname{div}\left(A^{\prime} \nabla \phi_{R}\right)\right|
$$

By computation we have

$$
\begin{aligned}
& \nabla \phi_{R}=-\phi_{1}^{\prime}(\left.\frac{1}{R \Gamma}\right) \frac{\nabla \Gamma}{R \Gamma}, \quad L^{*}\left(\phi_{R}\right)=\operatorname{div}\left(A^{\prime} \frac{\nabla \Gamma}{R \Gamma} \phi_{1}^{\prime}\left(\frac{1}{R \Gamma}\right)\right)= \\
&=-\phi_{1}^{\prime}\left(\frac{1}{R \Gamma}\right) \frac{L^{*} \Gamma}{R \Gamma}+(\nabla \Gamma(y) \cdot A \nabla \Gamma(y))\left(\frac{\phi_{1}^{\prime \prime}(t)}{R^{2} \Gamma^{4}}+2 \frac{\phi_{1}^{\prime}(t)}{R \Gamma^{3}}\right)_{t=1 / R \Gamma} \\
&=0+\frac{(\nabla \Gamma(y) \cdot A \nabla \Gamma(y))}{R^{2} \Gamma^{4}}\left(\phi_{1}^{\prime \prime}(t)+2 \frac{\phi_{1}^{\prime}(t)}{t}\right)_{t=1 / R \Gamma}
\end{aligned}
$$

where we have used the fact that $\phi_{R}^{\prime}=0$ in a neighbourhood of $x$ and $L^{*}(\Gamma)=0$ away from $x$. Therefore we have,

$$
\begin{aligned}
\int_{\Omega_{2 R}(x)} \phi_{R} \nu d y & \leq \int_{\Omega_{2 R}(x) \backslash \Omega_{R}(x)}|u| \frac{(\nabla \Gamma \cdot A \nabla \Gamma)}{R^{2} \Gamma^{4}}\left|\phi_{1}^{\prime \prime}(t)+2 \frac{\phi_{1}^{\prime}(t)}{t}\right|_{t=1 / R \Gamma} d y \\
& \leq \frac{M}{R^{2}} \int_{\Omega_{2 R}(x) \backslash \Omega_{R}(x)}|u| \frac{(\nabla \Gamma \cdot A \nabla \Gamma)}{\Gamma^{4}} d y
\end{aligned}
$$

thereby completing the proof. 


\subsection{Ring condition (3.2) related to $\nabla u$}

The results of this subsection deal with some conditions on the gradient of $u$ assuring that some form of the ring condition as in Lemma (26) is satisfied.

Lemma 29. Assume that $\left|A^{\prime} \nabla \Gamma\right| \in L_{\text {loc }}^{1}\left(\mathbb{R}^{N}\right)$. Let $u \in \mathscr{C}^{2}\left(\mathbb{R}^{N}\right)$ be such that $-L(u) \geq 0$ and let $x \in \mathbb{R}^{N}$. Then the following statements are equivalent.

1. There exists $l_{x} \in \mathbb{R}$ such that (3.50) holds that is

$$
\liminf _{R \rightarrow+\infty} \int_{\partial \Omega_{R}(x)} \frac{\left(\nabla \Gamma_{x} \cdot A \nabla \Gamma_{x}\right)}{\left|\nabla \Gamma_{x}\right|} u d H_{n-1}=l_{x} .
$$

2.

$$
\liminf _{r \rightarrow+\infty} \int_{\Omega_{r}(x)}\left(\nabla \Gamma_{x} \cdot A \nabla u\right) d y<+\infty
$$

3.

$$
\sup _{r>0} \int_{\Omega_{r}(x)}\left(\nabla \Gamma_{x} \cdot A \nabla u\right) d y<+\infty
$$

4. Let $D_{x}>2$ and set $g_{x}:=\Gamma_{x}^{\frac{1}{2-D_{x}}}$. Then

$$
\left(2-D_{x}\right) \int_{1}^{+\infty} \frac{d t}{t_{x}^{D}} \int_{g_{x}(y)<t}\left(\nabla g_{x} \cdot A \nabla u\right) d y<+\infty .
$$

5.

$$
\int_{1}^{+\infty} \frac{d t}{t^{2}} \int_{\partial \Omega_{t}(x)} \frac{\left(\nabla \Gamma_{x} \cdot A \nabla u\right)}{\left|\nabla \Gamma_{x}\right|}<+\infty
$$

6. Let $\sigma:=\sigma(x)>1$. Then

$$
\int_{1}^{+\infty} \frac{d t}{t^{\sigma}} \int_{\Omega_{t}(x)} \frac{\left(\nabla \Gamma_{x} \cdot A \nabla u\right)}{\Gamma_{x}^{\sigma-1}}<+\infty
$$

Moreover, if one of the above statements holds, then

$$
u(x)=l_{x}+\int_{\mathbb{R}^{N}} \Gamma(x, y) \nu(y) d y .
$$

Here $\nu$ is the Radon measure $\nu:=-L(u)$ and

$$
\liminf _{r \rightarrow+\infty} \int_{\Omega_{r}(x)}\left(\nabla \Gamma_{x} \cdot A \nabla u\right) d y=\int_{\mathbb{R}^{N}} \Gamma(x, y) \nu(y) d y .
$$

Remark 30. We notice that after combining the above lemma with what we have already proved and Theorem 6, we have now completed the proof of the Theorem 8 .

Before proving the lemma we state another result which seems to be of some interest in itself.

Proposition 31. Let $x \in \mathbb{R}^{N}$ and let $u \in \mathscr{C}^{2}\left(\mathbb{R}^{N}\right)$ be such that $-L(u) \geq 0$. Then

$$
\begin{gathered}
\int_{\partial \Omega_{r}(x)} \frac{\left(\nabla \Gamma_{x} \cdot A \nabla u\right)}{\left|\nabla \Gamma_{x}\right|} \geq 0, \quad \text { for a.e. } r>0 \\
\int_{\Omega_{r}(x) \backslash \Omega_{1}(x)} \frac{\left(\nabla \Gamma_{x} \cdot A \nabla u\right)}{\Gamma_{x}^{\gamma}} \geq 0 \quad \text { for any } r>1 \text { and } \gamma \in \mathbb{R} .
\end{gathered}
$$


Proof. Let $R>1$ and $\gamma \in \mathbb{R}$. Using coarea formula we have

$$
\begin{aligned}
\int_{1>\Gamma_{x}>\frac{1}{R}} \frac{\left(\nabla \Gamma_{x} \cdot A \nabla u\right)}{\Gamma_{x}^{\gamma}} & =\int_{1}^{R}\left(\int_{\partial \Omega_{r}(x)} \frac{\left(\nabla \Gamma_{x} \cdot A \nabla u\right)}{\Gamma_{x}^{\gamma-2}\left|\nabla \Gamma_{x}\right|}\right) d r \\
& =\int_{1}^{R} r^{\gamma-2}\left(\int_{\partial \Omega_{r}(x)} \frac{\left(\nabla \Gamma_{x} \cdot A \nabla u\right)}{\left|\nabla \Gamma_{x}\right|}\right) d r .
\end{aligned}
$$

In particular if $\gamma=0$, the above identity becomes

$$
M_{x}(R)-M_{x}(1)=\int_{1}^{R} r^{-2}\left(\int_{\partial \Omega_{r}(x)} \frac{\left(\nabla \Gamma_{x} \cdot A \nabla u\right)}{\left|\nabla \Gamma_{x}\right|}\right) d r .
$$

Since $M$ is nondecreasing with respect to $R$ it follows that its derivative is a.e. nonnegative, and (3.58) follows.

Finally, inequality (3.59) follows from (3.58) and (3.60).

Proof of Lemma 29 From Lemma 21, and Lemma 24 we know that for any $r>0$

$$
u(x)=N_{x}(r)+M_{x}(r),
$$

where $M_{x}(r)=\int_{\Omega_{r}(x)}\left(\nabla \Gamma_{x} \cdot A \nabla u\right) d y$.

Therefore the equivalence $1 . \Leftrightarrow 2$. easily follows.

The monotonicity of $M_{x}(r)$ gives the equivalence $2 . \Leftrightarrow 3$.

The equivalence 2 . $\Leftrightarrow 5$. follows directly from identity (3.62).

Since $M_{x}(r)$ is nondecreasing statement 3., can be rewritten as

$$
\lim _{r \rightarrow+\infty} \int_{\Omega_{r}(x) \backslash \Omega_{1}(x)}\left(\nabla \Gamma_{x} \cdot A \nabla u\right) d y<+\infty .
$$

Let $\sigma>1$. Observing that

$$
\frac{1}{s^{\sigma-1}}=\int_{s}^{+\infty} \frac{\sigma-1}{t^{\sigma}} d t
$$

we obtain

$$
\begin{aligned}
\int_{\Omega_{R}(x) \backslash \Omega_{1}(x)}\left(\nabla \Gamma_{x} \cdot A \nabla u\right) & =\int_{1>\Gamma_{x}>\frac{1}{R}}(1 / \Gamma)^{\sigma-1} \frac{\left(A \nabla \Gamma_{x} \cdot \nabla u\right)}{(1 / \Gamma)^{\sigma-1}} \\
& =\int_{1>\Gamma_{x}>\frac{1}{R}}(1 / \Gamma)^{\sigma-1}\left(\nabla \Gamma_{x} \cdot A \nabla u\right) \int_{1 / \Gamma}^{+\infty} \frac{\sigma-1}{t^{\sigma}} d t= \\
& =(\sigma-1) \int_{1}^{+\infty} \frac{d t}{t^{\sigma}}\left(\int_{1>\Gamma>\max \left(\frac{1}{t}, \frac{1}{R}\right)} \frac{\left(\nabla \Gamma_{x} \cdot A \nabla u\right)}{\Gamma^{\sigma-1}}\right) \\
& =(\sigma-1) \int_{1}^{+\infty} \frac{d t}{t^{\sigma}}\left(\int_{\Omega_{t} \cap \Omega_{R} \backslash \Omega_{1}} \frac{\left(\nabla \Gamma_{x} \cdot A \nabla u\right)}{\Gamma^{\sigma-1}}\right) .
\end{aligned}
$$

Since the integrand of

$$
\int_{\Omega_{t} \cap \Omega_{R} \backslash \Omega_{1}} \frac{\left(\nabla \Gamma_{x} \cdot A \nabla u\right)}{\Gamma^{\sigma-1}} d y
$$

is nonnegative we can pass to the limit as $R \rightarrow+\infty$, and by using Beppo Levi theorem the equivalence 3 . $\Leftrightarrow 6$. follows.

Hence the equivalence 3 . $\Leftrightarrow 4$. is proved.

The equivalence 4 . $\Leftrightarrow 6$. follows by the substitution $\Gamma_{x}=g_{x}^{2-D_{x}}$ and a suitable $\sigma$. 


\subsection{Proof of Theorem 6}

We begin noticing that the proof of the representation (3.3) in Theorem 6 follows from Lemma 26.

It remains to prove the others claims of the statement.

Proof. [ Aa] First we prove that inf $l \geq \inf u$. Without loss of generality we assume that $\inf u>-\infty$. From the ring condition 3.2 we have

$$
\begin{aligned}
& l(x)=\frac{1}{\ln 2} \liminf _{R \rightarrow \infty} \int_{\Omega_{2 R}}(x) \backslash \Omega_{R}(x) \frac{\left(\nabla \Gamma_{x} \cdot A \nabla \Gamma_{x}\right)}{\Gamma_{x}} u d y \geq \\
& \geq \frac{1}{\ln 2} \liminf _{R \rightarrow \infty} \int_{\Omega_{2 R}(x) \backslash \Omega_{R}(x)} \frac{\left(\nabla \Gamma_{x} \cdot A \nabla \Gamma_{x}\right)}{\Gamma_{x}} \inf u d y=\inf u .
\end{aligned}
$$

Hence $\inf l \geq \inf u$.

Next, since $u(x) \geq l(x)$, let $\left(x_{n}\right)$ be a minimising sequence of $u$. From the inequality

$$
u\left(x_{n}\right) \geq l\left(x_{n}\right) \geq \inf u,
$$

the claim follows.

Analogously we obtain the estimate of sup.

The alternative (3.4) follows directly from (3.3).

Proof. [Ab.] The first claim follows from Lemma 25.

In order to prove (3.5), we set $v(z):=u(z)-l(x)$ therefore $v$ is a distributional positive solution of $-L(v)=\nu$. Therefore from the estimate (3.57) (see below Theorem 28), for $R$ large we have

$$
\int_{\Omega_{s}(0)} \nu(y) d y \leq \int_{\Omega_{R}(x)} \nu(y) d y \leq 8 M R \int_{\Omega_{2 R}(x) \backslash \Omega_{R}(x)} v(y) \frac{\left(\nabla \Gamma_{x}(y) \cdot A(y) \nabla \Gamma_{x}(y)\right)}{\Gamma_{x}(y)} d y,
$$

and hence the claim follows.

Proof. [Ac.] The function $w$ is finite on the whole space, and it is measurable. Therefore $l$ is measurable too. Since $l$ and $w$ are nonnegative and their sum is the regular function $u$, it follows that they belong $L_{l o c}^{1}\left(\mathbb{R}^{N}\right)$.

Proof. [Ad] It follows directly from (3.3).

Proof. [B] Without loss of generality we can assume that $u$ is nonnegative.

From Lemma 24, it follows that the function $N(r)$ is nonincreasing and since the integrand is nonnegative, $N$ admits a finite nonnegative limit (as $r \rightarrow+\infty$ ), that is (3.51) is fulfilled and by Lemma 26, we get the claim.

Proof. [C] Set

$$
\mathcal{K}_{x}:=\frac{1}{\ln 2} \frac{\left(\nabla \Gamma_{x} \cdot A \nabla \Gamma_{x}\right)}{\Gamma_{x}}, \quad \text { and } \quad A_{R}(x):=\Omega_{2 R}(x) \backslash \Omega_{R}(x) .
$$

Let $w(x):=\int_{\mathbb{R}^{N}} \Gamma(x, y) \nu(y) d y$. Since $w \in \mathscr{C}^{2}\left(\mathbb{R}^{N}\right)$ is nonnegative and $-L(w)=\nu$ from $\mathrm{B}$ of Theorem 6 it follows that for $w$ satisfying (3.8) and

$$
w(x)=h_{1}(x)+\int_{\mathbb{R}^{N}} \Gamma(x, y) \nu(y) d y
$$

we have $h_{1}(x) \equiv 0$. On the other hand, $h_{1}$ is the harmonic function given by

$$
h_{1}(x)=\liminf _{R \rightarrow \infty} \int_{A_{R}(x)} \mathcal{K}_{x} w d y .
$$


Hence,

$$
\liminf _{R \rightarrow \infty} \int_{A_{R}(x)} \mathcal{K}_{x} w d y=0 \quad \forall x \in \mathbb{R}^{N} .
$$

Therefore, from the decomposition of $u$, by using (3.44) for the harmonic function $h$ we have

$$
\int_{A_{R}(x)} \mathcal{K}_{x} u d y=\int_{A_{R}(x)} \mathcal{K}_{x} h d y+\int_{A_{R}(x)} \mathcal{K}_{x} w d y=h(x)+\int_{A_{R}(x)} \mathcal{K}_{x} w d y .
$$

By taking the limit as $R \rightarrow \infty$ we get the claim.

\subsection{Proof of Theorems 14 and 19}

Theorem 14 can be seen as a particular case of Theorem 19 by taking $X \equiv \mathscr{C}^{2}\left(\mathbb{R}^{N}\right)$. So we shall present the proof for functions belonging to $\mathscr{C}^{2}\left(\mathbb{R}^{N}\right) \cap X$.

The implications $1 . \Rightarrow 2$. and $3 . \Rightarrow 4$. are immediate.

2 . $\Rightarrow 3$. Let $u \in \mathscr{C}^{2}\left(\mathbb{R}^{N}\right) \cap X$ be such that $-L(u)=\nu \geq 0$. Let $l \in \mathbb{R}$ and assume that the ring condition (3.12) holds. We have to prove that inf $u=l$.

Since the (3.12) holds for any $x \in \mathbb{R}$, we are in the position to apply point A of Theorem 6 and from point Aa of the same theorem, we have that inf $u=\inf l_{x}=l$.

To prove the converse implication, assume that inf $u=l \in \mathbb{R}$, we have to prove (3.12). Since $u$ is bounded from below, from B of Theorem 6 it follows that the ring condition (3.2) holds for any $x$. Now our claim is to prove such a function $l(x)$ is constant.

From Ac and Ab of Theorem 6 we deduce that $l(x)$ is a distributional solution of $L(h)=$ 0 , and hence by hypothesis it results that $l(x)$ is smooth. From (3.3) we get $u \geq l$ which implies that $l \in X$. From the Hypothesis 2., we have that $l(x)=l$ is constant, that is the claim.

4. $\Rightarrow 1$. Let $u \in \mathscr{C}^{2}\left(\mathbb{R}^{N}\right) \cap X$. Assume that $u$ can be represented as in (3.11), we have to prove that $-L(u)=\nu$ and $\inf u=m$. From Lemma 25 we have that $u$ is a solution of $-L(u)=\nu$. By hypothesis 4., the ring condition is satisfied with a constant function $c_{x}=c$. Therefore, the remaining claim follows from Theorem 6 .

We prove the converse implication. Let $u \in \mathscr{C}^{2}\left(\mathbb{R}^{N}\right) \cap X$ be such that $-L(u)=\nu \geq 0$ and $\inf u=m \in \mathbb{R}$ : we have to prove that (3.11) holds. Since $u$ is bounded from below, from hypothesis 4 . for $u$ the ring condition (3.2) holds with a constant function. The conclusion follows applying Theorem 6 .

Remark 32. We point out that the proof of Theorem 14 depends on the validity of Lemma 21. Consequently a version of Theorem 14 for distributional solutions $u \in L_{l o c}^{1}\left(\mathbb{R}^{N}\right)$ holds provided (3.25) and (3.26) are satisfied in the $L_{l o c}^{1}\left(\mathbb{R}^{N}\right)$ setting and $-L(u) \geq 0$. We do not know if this is possible in the general framework of section 2.

In the context of homogeneous Carnot group this is possible as we shall see in the following section 4 .

\subsection{Additional remarks on the ring conditions}

In this section we show some cases when the ring conditions are satisfied.

\subsubsection{Ring condition and semilinear problems}

In this section we investigate the connection between the ring condition and the solution of the inequality

$$
-L(u) \geq|u|^{q} \quad \text { on } \mathbb{R}^{N} \text {. }
$$


As in Section 3.6.1, here we do not make any assumption on the sign of the solution $u$ and from the universal esimate in Section 3.6.1 we derive that, in some cases the ring conditions (3.2) and (3.48) hold.

For several reasons that will be clarified at the end of this section, we shall deal with a more general inequalities involving a bounded coefficent $a$ (see (3.65) below).

In order to formulate the results, we shall use the same notation of Remark 20, that is, fix $x \in \mathbb{R}^{N}$ and choose a real number $D_{x}>2$. Next we define $g_{x}(y):=\Gamma_{x}^{\frac{1}{2-D_{x}}}(y)$. The function $g_{x}: \mathbb{R}^{N} \rightarrow \mathbb{R}$ is continuous, positive and smooth in $\mathbb{R}^{N} \backslash\{x\}$ and $g_{x}(x)=0$. We set $h_{x}^{2}:=\left(\nabla g_{x} \cdot A \nabla g_{x}\right)$.

Theorem 33. Let $q>1, a \in L^{\infty}\left(\mathbb{R}^{N}\right)$ and $x \in \mathbb{R}^{N}$. There exists a constant $C=C(x)>0$ such that if $u \in C^{2}\left(\mathbb{R}^{N}\right)$ is a solution of

$$
-L(a u) \geq h_{x}^{2}|u|^{q} \quad \text { on } \mathbb{R}^{N}
$$

then for any $R>0$ we have,

$$
\int_{\Omega_{R}(x)} h_{x}^{2}|u|^{q} d y \leq\|a\|_{\infty} C R^{\frac{D_{x}-2 q^{\prime}}{D_{x}-2}}
$$

where $C=C_{1}(q)\left(D_{x}-2\right)^{\frac{2}{q-1}}$. Moreover the following chain of inequalities hold,

$$
\begin{gathered}
\int_{\Omega_{2 R}(x) \backslash \Omega_{R}(x)} \frac{\left(\nabla \Gamma_{x} \cdot A \nabla \Gamma_{x}\right)}{\Gamma_{x}}|a u| d y \leq \\
c \int_{\Omega_{2 R}(x) \backslash \Omega_{R}(x)} \frac{\left(\nabla \Gamma_{x} \cdot A \nabla \Gamma_{x}\right)}{\Gamma_{x}}|u| d y \leq \\
c\left(\int_{\Omega_{2 R}(x) \backslash \Omega_{R}(x)} \frac{\left(\nabla \Gamma_{x} \cdot A \nabla \Gamma_{x}\right)}{\Gamma_{x}}|u|^{q} d y\right)^{1 / q} \leq C R^{-\frac{2}{q-1} \frac{1}{D_{x}-2}} .
\end{gathered}
$$

Therefore, if au is smooth, we have

$$
a(x) u(x) \geq \int_{\mathbb{R}^{N}} \Gamma(x, y) h_{x}(y)|u(y)|^{q} d y .
$$

In particular, if

$$
h_{x} \text { is bounded on } \mathbb{R}^{N} \backslash \Omega_{S}(x) \text { for a large } S \text {, }
$$

and for a positive constant $c>0, u$ solves also

$$
-L(u) \geq c|u|^{q} \quad \text { on } \mathbb{R}^{N},
$$

then

$$
\int_{\Omega_{R}(x)}|u|^{q} d y \leq C R^{\frac{D_{x}-2 q^{\prime}}{D_{x}-2}}, \quad \text { for } R>S .
$$

Proof. From Theorem 28, with $\nu=h_{x}^{2}|u|^{q}$, we have

$$
\begin{gathered}
\int_{\Omega_{2 R}(x)} h_{x}^{2}|u|^{q} \phi_{R} d y \leq 8 M R\|a\|_{\infty} \int_{\Omega_{2 R}(x) \backslash \Omega_{R}(x)}|u| \phi_{R}^{1 / q} \frac{(\nabla \Gamma \cdot A \nabla \Gamma)}{\Gamma} d y \\
=c R \int_{\Omega_{2 R} \backslash \Omega_{R}(x)}|u| \phi_{R}^{1 / q} h_{x}^{2} \Gamma^{\frac{D_{x}}{D_{x}-2}} d y .
\end{gathered}
$$


Using Hölder inequality we obtain

$$
\int_{\Omega_{2 R}} h_{x}^{2}|u|^{q} \phi_{R} \leq c R\left(\int_{\Omega_{2 R}(x) \backslash \Omega_{R}} h_{x}^{2}|u|^{q} \phi_{R}\right)^{1 / q}\left(\int_{\Omega_{2 R}(x) \backslash \Omega_{R}} h_{x}^{2} \Gamma^{\frac{D_{x}}{D_{x}-2} q^{\prime}}\right)^{1 / q^{\prime}} d y
$$

which implies,

$$
\begin{array}{r}
\int_{\Omega_{R}(x)} h_{x}^{2}|u|^{q} d y \leq \int_{\Omega_{2 R}(x)} h_{x}^{2}|u|^{q} \phi_{R} d y \leq c R^{q^{\prime}} \int_{\Omega_{2 R}(x) \backslash \Omega_{R}(x)} h_{x}^{2} \Gamma^{\frac{D_{x}}{D_{x}-2}} \Gamma^{\frac{D_{x}}{D_{x}-2}\left(q^{\prime}-1\right)} d y \\
\leq c R^{\frac{D_{x}-2 q^{\prime}}{D_{x}-2}} \int_{\Omega_{2 R}(x) \backslash \Omega_{R}(x)}^{\Gamma^{\frac{D_{x}}{D_{x}-2}} h_{x}^{2} d y \leq c R^{\frac{D_{x}-2 q^{\prime}}{D_{x}-2}} .}
\end{array}
$$

Here we have used the fact that if $y \in \Omega_{2 R}(x) \backslash \Omega_{R}(x)$ then $\Gamma(y) \leq \frac{1}{R}$ and (3.20) holds.

The chain of inequalities follow directly from Hölder inequalities, (3.20) and (3.67).

A slight modification of the above argument proves the estimate (3.74).

An immediate application of above result yields several Liouville type theorems for semilinear elliptic inequalities as well as qualitative information on the solutions. We refer the interested reader to [13].

Here for sake of brevity, we point out a simple example.

First of all from (3.71) we immediately get the information that if $u \in C^{2}\left(\mathbb{R}^{N}\right)$ is non trivial solution of (3.66) with $a \in L^{\infty}\left(\mathbb{R}^{N}\right) \cap \mathscr{C}^{2}\left(\mathbb{R}^{N}\right)$ then $a(x) u(x)>0$. Therefore if the weight $a$ vanishes in one point $x$ then (3.66) has no nontrivial solution (without any assumption on the sign).

Looking at "coercive" inequality

$$
L(u) \geq h_{x}^{2}|u|^{q} \quad \text { on } \mathbb{R}^{N},
$$

from (3.71) it follows that either $u(x)<0$ or $u \equiv 0$. Hence we can state the following.

Theorem 34. Let $q>1$ and $u \in \mathscr{C}^{2}\left(\mathbb{R}^{N}\right)$ be a solution of the equation

$$
L(u)=|u|^{q-1} u \quad \text { on } \mathbb{R}^{N} .
$$

If for some point $x \in \mathbb{R}^{N}$ there exists $D_{x}>2$ such that the function $h_{x}^{2}$ (as defined at the beginning of this section and in Remark 20) satisfies (3.72), then $u \equiv 0$.

Proof. By computation we have that $u^{2}$ satisfies

$$
L\left(u^{2}\right)=2(A \nabla u \cdot \nabla u)+2 u L(u)=2(A \nabla u \cdot \nabla u)+2|u|^{q+1} \geq 2|u|^{q+1} \geq c h_{x}^{2}|u|^{q+1} .
$$

Therefore, as observed before, $u^{2}(x)<0$ or $u^{2} \equiv 0$, which concludes the proof.

The above results when $L=\Delta$ in the Euclidean space and for distributional solution has been proved by Brezis in [6]. While for distributional solutions in the Carnot group setting and for sub-Laplacians see Corollary 4.5 of the author's paper [12]. See also [11] for some quasilinear generalisations.

\subsubsection{Other assumptions}

In this section we shall present some condition on $u$ that assure the validity of the ring conditions (3.2) and (3.48) with $l_{x}=0$. 
We recall that for a given positive measure $\mu$ on an open set $U$ and $q>1$ the weak- $L^{q}$ space is set $L_{w}^{q}(U, \mu)$ of the functions $f \in L_{l o c}^{1}(U)$ such that

$$
\sup _{\mu(A)<\infty}(\mu(A))^{-1 / q^{\prime}} \int_{A}|f(y)| d \mu(y)<+\infty,
$$

here $A$ is a measurable set contained in $U$.

Theorem 35. Let $x \in \mathbb{R}^{N}$ be fixed. In what follows $\mu_{x}$ shall denote the measure $h_{x}^{2}(y) d y$ or if (3.72) holds $\mu_{x}$ stands for the Lebesgue measure.

Assume that $u \in L_{w}^{q}\left(\mathbb{R}^{N} \backslash \Omega_{S}(x), \mu_{x}\right)$ for $S$ large and $q>1$. Then u satisfies (3.48) with $l_{x}=0$.

In addition, if $u \in \mathscr{C}^{2}\left(\mathbb{R}^{N}\right)$ and $-L(u) \geq 0$ with $1<q<D_{x} /\left(D_{x}-2\right)$ then $u \equiv 0$.

The threshold $D_{x} /\left(D_{x}-2\right)$ is sharp. Indeed in the Euclidean setting there exist positive superharmonic function in $L_{w}^{q}\left(\mathbb{R}^{N}\right)$ with $q \geq N /(N-2)$.

Before proving the theorem, we notice that from Lemma 23 we have that

$$
\mu_{x}\left(\Omega_{2 R}(x) \backslash \Omega_{R}(x)\right) \approx R^{\frac{D x}{D_{x}-2}} \quad \text { for } \quad R>0 .
$$

Proof. Set $A_{R}:=\Omega_{2 R}(x) \backslash \Omega_{R}(x)$. Taking into account that $u \in L_{w}^{q}\left(\mathbb{R}^{N} \backslash \Omega_{S}(x), \mu_{x}\right)$ and the estimate on $\mu_{x}\left(A_{R}\right)$, we have

$$
\int_{A_{R}} \frac{\left(\nabla \Gamma_{x} \cdot A \nabla \Gamma_{x}\right)}{\Gamma_{x}}|u| d y \approx \frac{1}{R^{\frac{D_{x}}{D_{x}-2}}} \int_{A_{R}}|u| d \mu \leq c \frac{1}{R^{\frac{D_{x}}{D_{x}-2}}} \mu_{x}\left(A_{R}\right)^{1 / q^{\prime}} \leq c R^{-\frac{1}{q} \frac{D_{x}}{D_{x}-2}} .
$$

Letting $R \rightarrow+\infty$, we get the claim.

The second part of the theorem follows from Theorem 6 . Indeed $u$ can be represented as in (3.3) with $l_{x}=0$. If $1<q<D_{x} /\left(D_{x}-2\right)$, then from (3.76) it follows that,

$$
\lim _{R \rightarrow+\infty} R \int_{A_{R}} \frac{\left(\nabla \Gamma_{x} \cdot A \nabla \Gamma_{x}\right)}{\Gamma_{x}}|u| d y=0 .
$$

This contradicts (3.5).

\section{Applications in the Carnot groups setting}

In the setting of Carnot groups the previous results can be extended to distributional solutions. The main ingredient for this goal is a regularisation procedure by a family of mollifiers that commutes with the sub-Laplacian. See [5].

Therefore all the result in previous section can be restated for distributional solutions and the representation formula hold almost everywhere. For the convenience of the reader we shall rewrite them here. We state the results in terms of the balls $B_{N_{2}}$ generated by the gauge $N_{2}$ instead of $\Omega_{r}(x)$.

Lemma 36. Let $u \in L_{l o c}^{1}\left(\mathbb{R}^{N}\right)$ be weakly superharmonic and let $\nu$ be its Radon measure, that is $-\Delta_{G} u=\nu$ in distributional sense. Then, for any $\beta \neq 0, R>0,0<\delta<1$, and a.e. $x \in \mathbb{R}^{N}$ and a.e. $r>0$, the identities (3.32), (3.33), (3.34) in Remark 22 hold by replacing $D_{x}=Q, g_{x}(y)=N_{2}\left(x^{-1} \circ y\right)$, and $h_{x}^{2}=\phi_{x}^{2}$.

The first representation formula for distributional solutions in the Carnot group setting can be stated as follows. This is the analogue of theorem 6 . 
Theorem 37. Let $u \in L_{\text {loc }}^{1}\left(\mathbb{R}^{N}\right)$ be such that $-\Delta_{G} u=\nu \geq 0$. Let $x \in \mathbb{R}^{N}$ and $l_{x} \in \mathbb{R}$ be such that

$$
\left.\liminf _{R \rightarrow+\infty} \int_{B_{N_{2}}(x, 2 R) \backslash B_{N_{2}}(x, R)} \frac{\psi_{0}^{2}}{N_{2}^{Q}}\left(x^{-1} \circ y\right)\right)\left(u(y)-l_{x}\right) d y=0 .
$$

Then if $x$ is a Lebesgue point for $u$ we have

$$
u(x)=l_{x}+\int_{\mathbb{R}^{N}} \frac{d \nu(y)}{N_{2}^{Q-2}\left(x^{-1} \circ y\right)} .
$$

In particular, if (4.1) holds for a.e. $x \in \mathbb{R}^{N}$, and $l_{x}=l$ does not depend on $x$, then $l=\operatorname{essinf}_{\mathbb{R}^{N}} u \in \mathbb{R}$ and

$$
u(x)=l+\int_{\mathbb{R}^{N}} \Gamma(x, y) \nu(y) d y . \quad \text { a.e. } x \in \mathbb{R}^{N} .
$$

Similarly Theorem 14, can be formulated as follows.

Theorem 38. The following statements are equivalent.

1. Let $u \in L_{\text {loc }}^{1}\left(\mathbb{R}^{N}\right)$ be such that $-\Delta_{G} u=\nu \geq 0$ and $\operatorname{ess}_{\inf } \mathbb{R}^{N} u=l \in \mathbb{R}$. Then

$$
u(x)=l+\int_{\mathbb{R}^{N}} \frac{d \nu(y)}{N_{2}^{Q-2}\left(x^{-1} \circ y\right)} \quad \text { a.e. } x \in \mathbb{R}^{N} .
$$

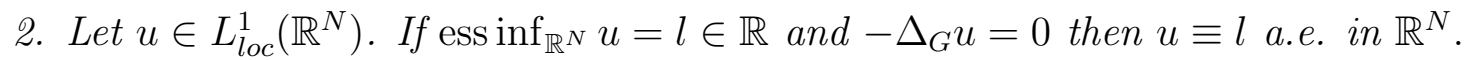

3. Let $u \in L_{l o c}^{1}\left(\mathbb{R}^{N}\right)$ be such that $-\Delta_{G} u=\nu \geq 0$. Then, $\operatorname{ess} \inf _{\mathbb{R}^{N}} u=l \in \mathbb{R}$ if and only if

$$
\liminf _{R \rightarrow+\infty} f_{B_{N_{2}}(x, 2 R) \backslash B_{N_{2}}(x, R)} \psi_{x}^{2}(u-l) d y=0 \quad \text { a.e. } x \in \mathbb{R}^{N}
$$

if and only if

$$
\lim _{R \rightarrow+\infty} f_{B_{N_{2}}(x, 2 R) \backslash B_{N_{2}}(x, R)} \psi_{x}^{2}|u-l| d y=0 \quad \text { a.e. } x \in \mathbb{R}^{N} .
$$

Remark 39. In the Carnot group setting a classical Liouville theorem has been proved in [4] for distributional solutions. See also [5]. Therefore the representation formula holds and the infimum of superharmonic function can be characterised by (4.5) and (4.6).

In the same framework a proof of the representation formula based on harmonic analysis argument is contained in the book [5] under the assumption that $u$ is bounded from below. Notice that in [15] the authors prove the first implication of Theorem 37. That is if $u \in$ $L_{l o c}^{1}\left(\mathbb{R}^{N}\right)$ is a distributional solution of $-\Delta_{G} u=f \geq 0$ and satisfies (4.6) then

$$
u(x)=l+\int_{\mathbb{R}^{N}} \Gamma(x, y) f(y) d y,
$$

a.e. on $\mathbb{R}^{N}$. Finally we want to point out that if $u \in L_{w}^{q}\left(\mathbb{R}^{N}\right)$ for some $q>1$ then $u$ satisfies (4.6) with $l=0$.

\section{Acknowledgement}

We warmly thank Professor Ermanno Lanconelli for useful comments and remarks, and for pointing out to us the reference [1]. 


\section{References}

[1] B. AbBondanza, Potential Analysis for Hypoelliptic Second Order PDEs with Nonnegative Characteristic Form, Tesi di Dottorato, Università di Bologna, (2015), 1-129.

[2] W.Bauer, K. Furutani, C. IWAsaki, Fundamental solution of a higher step Grushin type operator, Adv. Math. 271 (2015), 188-234.

[3] S. Biagi, A. Bonfiglioli, The existence of a global fundamental solution for homogeneous Hörmander operators via a global lifting method, Proc. London Math. Soc. 114 (2017) 855-889.

[4] A. Bonfiglioli, E. Lanconelli, Liouville-type theorems for real sub-Laplacians, Manuscripta Math. 105 (2001), no. 1, 111-124.

[5] A. Bonfiglioli, E. Lanconelli, F. Uguzzoni, Stratified Lie Groups and Potential Theory for their sub-Laplacians, Springer, 2007.

[6] H. Brezis, Semilinear equations in $\mathbb{R}^{n}$ without condition at infinity, Appl. Math. Optimization, 12 (1984), 271-282.

[7] G. Caristi, L. D’Ambrosio and E. Mitidieri, Liouville Theorems for Some Nonlinear Inequalities, Proc. Steklov Inst. Math., 260, (2008) 90-111.

[8] G. Caristi, L. D'Ambrosio And E. Mitidieri, Liouville theorems and representation formulae for higher order problems and systems and related Liouville theorems, Milan J. Math. 76 (2008), 27-67.

[9] G. Citti, N. Garofalo and E. Lanconelli, Harnack's inequality for sum of squares of vector fields plus a potential, Amer. J. Math., 115, (1993), 699-734.

[10] L. D'Ambrosio And E. Mitidieri, Nonnegative solutions of some quasilinear elliptic inequalities and applications, SB MATH, 201(6), (2010) 855-871.

[11] L. D'Ambrosio And E. Mitidieri, A priori estimates and reduction principles for quasilinear elliptic problems and applications, Adv. Diff.Eq., 17, 9-10, (2012) 935-1000.

[12] L. D'Ambrosio And E. Mitidieri, Uniqueness and comparison principles for semilinear equations and inequalities in Carnot groups, Adv. Nonlinear Anal. 7(3), (2018) 313-325.

[13] L. D'Ambrosio And E. Mitidieri, Liouville Theorems of some second order elliptic inequalities, Preprint, (2018) 1-40.

[14] L. D'Ambrosio And E. Mitidieri, A priori estimates, positivity results, and nonexistence theorems for quasilinear degenerate elliptic inequalities Adv. Math., 224 (2010) 967-1020.

[15] L. D'Ambrosio, E. Mitidieri And S. I. Pohozaev, Representation formulae and inequalities for solutions of a class of second order partial differential equations, Trans. Amer. Math. Soc., 358 (2006), 893-910.

[16] G.B. Folland, Subelliptic estimates and function spaces on nilpotent Lie groups, Arkiv gör Mat. 13 (1975), 161-207.

[17] G.B. Folland, E.M. Stein, Hardy spaces on Homogeneous Groups, Math. Notes, vol. 28, Princeton Univ. Press, Princeton, 1982.

[18] P.C. Greiner, A fundamental solution for a nonelliptic partial differential operator, Can. J. Math. 31 (1979), 1107-1120. 
[19] J. Heinonen, Calculus on Carnot Groups, in Fall School in Analysis (Jyväskylä, 1994), vol. 68, Ber. Univ. Jyväskylä Math. Inst. Jyväskylä, 1995, 1-31.

[20] E. H. Lieb And M. Loss, Analysis, Graduate Studies in Mathematics Vol. 14, AMS, 2001.

[21] E. Mitidieri And S.I. Pohozaev, A priori estimates and the absence of solutions of nonlinear partial differential equations and inequalities, Proc. Steklov Inst. Math. 234 (2001), 1-362.

[22] E. Mitidieri And S.I. Pohozaev, Positivity property of solutions of some elliptic inequalities on $\mathbb{R}^{n}$, Doklady Math. 68 (2003), 339-344.

[23] SÁnchez-Calle, Antonio, Fundamental solutions and geometry of the sum of squares of vector fields, Invent. Math. 78 (1984), 143-160.

(1) Lorenzo D'Ambrosio

Dipartimento di Matematica

Università degli Studi di Bari

via E.Orabona, 4, I-70125 Bari

e-mail: dambros@dm.uniba.it

(2)

Enzo MitidieRI

Dipartimento di Matematica e Geoscienze

Università degli Studi di Trieste

via A.Valerio, 12/1, I-34127 Trieste

e-mail: mitidier@units.it 